Pacific

Journal of

Mathematics

LEFSCHETZ NUMBERS OF SYMPLECTIC INVOLUTIONS ON ARITHMETIC GROUPS

STEFFEN KIONKE

Volume $271 \quad$ No. 2

October 2014 


\title{
LEFSCHETZ NUMBERS OF SYMPLECTIC INVOLUTIONS ON ARITHMETIC GROUPS
}

\author{
STEFFEN KIONKE
}

\begin{abstract}
The reduced norm-one group $G$ of a central simple algebra is an inner form of the special linear group, and an involution on the algebra induces an automorphism of $G$. We study the action of such automorphisms in the cohomology of arithmetic subgroups of $G$. The main result is a precise formula for Lefschetz numbers of automorphisms induced by involutions of symplectic type. Our approach is based on a careful study of the smoothness properties of group schemes associated with orders in central simple algebras. Along the way we also derive an adelic reformulation of Harder's Gauss-Bonnet theorem.
\end{abstract}

\section{Introduction}

Let $G$ be a semisimple linear algebraic group defined over the field $\mathbb{Q}$ of rational numbers. Given a torsion-free arithmetic subgroup $\Gamma \subset G(\mathbb{Q})$, it is in general a very difficult task to compute the (cohomological) Betti numbers of $\Gamma$. However Harder's Gauss-Bonnet theorem [Harder 1971] makes it possible to determine the Euler characteristic of arithmetic groups. If the Euler characteristic is nonzero, one can extract information on the Betti numbers. Moreover, whether or not the Euler characteristic vanishes only depends on the structure of the associated real Lie group $G(\mathbb{R})$ (see the remark on page 384 ). If the Euler characteristic vanishes, Lefschetz numbers of automorphisms of finite order of $G$ are a suitable substitute to gain insight into the cohomology of $\Gamma$. The idea to study Lefschetz numbers in the cohomology of arithmetic groups goes back to Harder [1975]. A general method was developed by J. Rohlfs, first for Galois automorphisms [1978] and later in a general adelic setting [1990]. Lefschetz numbers were also studied in [Lee and Schwermer 1983; Lai 1991]. However, only very few groups have been considered in detail; most frequently Lefschetz numbers on Bianchi groups have been studied (see [Krämer 1985; Rohlfs 1985; Sengün and Türkelli 2012; Kionke and Schwermer 2012]). In this article we describe a method (based on Rohlfs'

The author was supported by FWF Austrian Science Fund, grant P 21090-N13.

MSC2010: primary 11F75; secondary 20H10, $20 \mathrm{G} 35$.

Keywords: arithmetic group, cohomology, Lefschetz number, involution. 
approach) to compute Lefschetz numbers of specific automorphisms on arithmetic subgroups of inner forms of the special linear group.

More precisely, let $F$ be an algebraic number field and let $A$ be a central simple $F$-algebra. The reduced norm $\operatorname{nrd}_{A / F}$ is a polynomial function on $A$ and the associated reduced norm-one group $G=\mathrm{SL}_{A}$ is a linear algebraic group defined over $F$. Indeed, the algebraic group $G$ is an inner form of the special linear group. If $A$ has an involution $\sigma$ of symplectic type (see the definition on page 377), then the composition of $\sigma$ with the group inversion yields an automorphism $\sigma^{*}$ of $G$. We study the Lefschetz numbers of such automorphisms induced by involutions of symplectic type.

1A. The main result. Let $F$ be an algebraic number field and let $O$ denote its ring of integers. Let $A$ be a central simple $F$-algebra. For our purposes we may assume that $A=M_{n}(D)$ for some quaternion $F$-algebra $D$ (see Section 1C).

Let $\Lambda_{D} \subseteq D$ be a maximal 0 -order in $D$; then $\Lambda:=M_{n}\left(\Lambda_{D}\right)$ is a maximal $\mathcal{O}$-order in $A$. For a nontrivial ideal $\mathfrak{a} \subseteq \mathcal{O}$ we study the cohomology of the principal congruence subgroups

$$
\Gamma(\mathfrak{a}):=\left\{g \in M_{n}\left(\Lambda_{D}\right) \mid \operatorname{nrd}_{A}(g)=1 \text { and } g \equiv 1 \bmod \mathfrak{a}\right\}
$$

of $G$. In fact, for $n \geq 2$ the groups $\Gamma(\mathfrak{a})$ have vanishing Euler characteristic.

The quaternion algebra $D$ is equipped with a unique involution of symplectic type $\tau_{c}: D \rightarrow D$, called conjugation, which induces an involution of symplectic type $\tau: A \rightarrow A$ by $\tau(x):=\tau_{c}(x)^{T}$; that is, apply $\tau_{c}$ to every entry of the matrix and then transpose the matrix. We will call $\tau$ the standard involution of symplectic type on $M_{n}(D)$. Composition of $\tau$ with the group inversion yields an automorphism $\tau^{*}$ of order two on $G$. Note that the congruence groups $\Gamma(\mathfrak{a})$ are stable under $\tau^{*}$. Fix a rational representation $\rho: G \times{ }_{F} \bar{F} \rightarrow \mathrm{GL}(W)$ of $G$ (defined over the algebraic closure of $F$ ) on a finite dimensional vector space. If $W$ is equipped with a compatible $\tau^{*}$-action (see the definition on page 389), then we can define the Lefschetz number $\mathscr{L}\left(\tau^{*}, \Gamma(\mathfrak{a}), W\right)$ of $\tau^{*}$ in the cohomology $H^{*}(\Gamma(\mathfrak{a}), W)$.

Main Theorem. Assume that $\Gamma(\mathfrak{a})$ is torsion-free. If $D$ is totally definite, we assume further that $n \geq 2$. The Lefschetz number $\mathscr{L}\left(\tau^{*}, \Gamma(\mathfrak{a}), W\right)$ is zero if $F$ is not totally real.

If $F$ is totally real, the Lefschetz number is given by the formula

$$
\mathscr{L}\left(\tau^{*}, \Gamma(\mathfrak{a}), W\right)=2^{-r} \mathrm{~N}(\mathfrak{a})^{n(2 n+1)} \Delta_{\mathrm{rd}}(D)^{n(n+1) / 2} \operatorname{Tr}\left(\tau^{*} \mid W\right) \prod_{j=1}^{n} M(j, \mathfrak{a}, D) .
$$

Here $\Delta_{\mathrm{rd}}(D)$ denotes the signed reduced discriminant of $D$ (see the definition on page 390), $r$ denotes the number of real places of $F$ ramified in $D$, and 


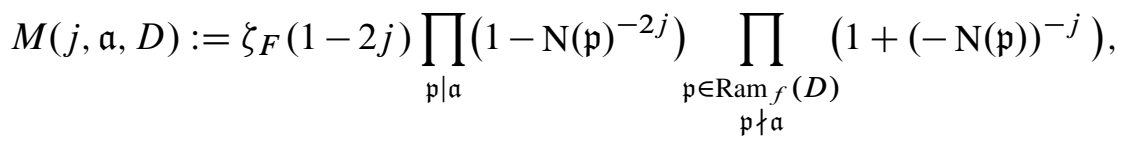

where $\operatorname{Ram}_{f}(D)$ denotes the set of finite places of $F$ where $D$ ramifies and $\zeta_{F}$ denotes the Dedekind zeta-function of $F$. If $F$ is totally real, then the Lefschetz number is zero if and only if $\operatorname{Tr}\left(\tau^{*} \mid W\right)=0$.

1B. Applications. We briefly give three applications of the above formula where we always assume $F$ to be a totally real number field.

1B1. Growth of the total Betti number. The analysis of the asymptotic behaviour of Betti numbers of arithmetic groups is an important topic. Calegari and Emerton [2009] have provided strong asymptotic upper bounds. We can use the main theorem to obtain an asymptotic lower bound result.

Let $G$ be the reduced norm-one group associated with the central simple $F$ algebra $M_{n}(D)$. For a torsion-free arithmetic subgroup $\Gamma \subseteq G(F)$ we define the total Betti number $B(\Gamma)$ as $\sum_{i=0}^{\infty} \operatorname{dim} H^{i}(\Gamma, \mathbb{C})$. Note that this is a finite sum since torsion-free arithmetic groups are of type (FL) (see [Borel and Serre 1973, Theorem 11.4.4]).

Corollary 1.1. Let $\Gamma_{0} \subset G(F)$ be an arithmetic subgroup. For any ideal $\mathfrak{a} \subset \mathbb{O}$ we define $\Gamma_{0}(\mathfrak{a}):=\Gamma_{0} \cap \Gamma(\mathfrak{a})$. There is a positive real number $\kappa>0$, depending on $F$, $D, \Gamma_{0}$, and $n$, such that

$$
B\left(\Gamma_{0}(\mathfrak{a})\right) \geq \kappa\left[\Gamma_{0}: \Gamma_{0}(\mathfrak{a})\right]^{\frac{n(2 n+1)}{4 n^{2}-1}}
$$

for every ideal $\mathfrak{a}$ such that $\Gamma(\mathfrak{a})$ is torsion-free.

A proof of this corollary will be given in Section 5E.

1B2. Rationality of zeta values. Note that the Lefschetz number is an integer since $\tau^{*}$ is of order two. We obtain a new proof of a classical theorem of Siegel [1969] and Klingen [1962].

Corollary 1.2. If $F$ is a totally real number field, then $\zeta_{F}(1-2 m)$ is a nonzero rational number for all integers $m \geq 1$.

Proof. Apply the main theorem with $D=M_{2}(F), \Lambda_{D}=M_{2}(\mathcal{O})$ and choose $W$ to be the trivial one-dimensional representation. We see that for every $n \geq 1$ and all sufficiently small ideals $\mathfrak{a} \subseteq \mathcal{O}$, the number

$$
\mathrm{N}(\mathfrak{a})^{n(2 n+1)} \prod_{j=1}^{n}\left(\zeta_{F}(1-2 j) \prod_{\mathfrak{p} \mid \mathfrak{a}}\left(1-\mathrm{N}(\mathfrak{p})^{-2 j}\right)\right)
$$

is a nonzero integer. The claim follows by induction on $m$. 
1B3. Cohomology of cocompact Fuchsian groups. Let $D$ be a division quaternion algebra over $F$ such that $D$ is split at precisely one real place $v_{0}$ of $F$. Therefore $r=[F: \mathbb{Q}]-1$ is the number of real places ramified in $D$.

Let $\Lambda=\Lambda_{D}$ be a maximal 0 -order in $D$. We consider the reduced norm-one group $G=\mathrm{SL}_{D}$ defined over $F$. The associated real Lie group is

$$
G_{\infty} \cong \mathrm{SL}_{2}(\mathbb{R}) \times \mathrm{SL}_{1}(\mathbb{H})^{r} .
$$

Note that the group $\mathrm{SL}_{1}(\mathbb{H})$ is compact and so the projection $p_{1}: G_{\infty} \rightarrow \mathrm{SL}_{2}(\mathbb{R})$ onto the first factor is a proper and open homomorphism of Lie groups. In particular, every discrete torsion-free subgroup $\Gamma \subseteq G_{\infty}$ maps via $p_{1}$ isomorphically to a discrete subgroup in $\mathrm{SL}_{2}(\mathbb{R})$.

Let $\mathfrak{a} \subseteq \mathcal{O}$ be a proper ideal such that $\Gamma(\mathfrak{a})$ is torsion-free. We will interpret $\Gamma(\mathfrak{a})$ as a subgroup of $\mathrm{SL}_{2}(\mathbb{R})$. Note that since we assumed $D$ to be a division algebra, the group $\Gamma(\mathfrak{a})$ is a cocompact Fuchsian group [Katok 1992, Theorem 5.4.1].

Let $\mathfrak{h}=\mathrm{SL}_{2}(\mathbb{R}) / \mathrm{SO}(2)$ be the Poincaré upper half-plane.

Corollary 1.3. The compact Riemann surface $\mathfrak{h} / \Gamma(\mathfrak{a})$ has genus

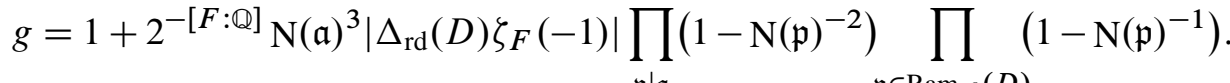

$$
\begin{aligned}
& \mathfrak{p} \mid \mathfrak{a} \\
& \mathfrak{p} \in \operatorname{Ram}_{\mathfrak{p} \nmid \mathfrak{a}}(D)
\end{aligned}
$$

This implies an explicit formula for the first Betti number $b_{1}(\Gamma(\mathfrak{a}))$ since

$$
b_{1}(\Gamma(\mathfrak{a}))=\operatorname{dim} H^{1}(\Gamma(\mathfrak{a}), \mathbb{C})=2 g .
$$

Proof. Consider the main theorem for $n=1$. Note that for $n=1$ the automorphism $\tau^{*}$ is actually the identity. This means that, using the main theorem with the trivial representation,

$$
\mathscr{L}\left(\tau^{*}, \Gamma(\mathfrak{a}), \mathbb{C}\right)=\chi(\Gamma(\mathfrak{a}))=\chi(\mathfrak{h} / \Gamma(\mathfrak{a})) .
$$

Note that the sign of the Lefschetz number is -1 . Since $\chi(\mathfrak{h} / \Gamma(\mathfrak{a}))=2-2 g$, the claim follows immediately.

In fact Corollary 1.3 yields a precise formula for the dimension of the space of holomorphic weight- $k$ modular forms for the group $\Gamma(\mathfrak{a})$ [Shimura 1971, Theorems 2.24 and 2.25].

1C. Reduction to quaternion algebras. Let $A$ be a central simple $F$-algebra. If $A$ has an involution $\sigma$ of symplectic type (see the definition on page 377), then $A$ is isomorphic to the opposed $F$-algebra $A^{\text {op }}$. This means that the class of $A$ has order two in the Brauer group of $F$. Since the dimension of $A$ is even, it follows from [Reiner 2003, Theorem (32.19)] that $A$ is isomorphic to a matrix algebra $M_{n}(D)$ over a quaternion algebra $D$. Therefore we always assume $A=M_{n}(D)$. 
Let $\tau$ be the standard involution of symplectic type on $M_{n}(D)$. Note that in this case $\sigma=\operatorname{int}(g) \circ \tau$ for an element $g \in \mathrm{GL}_{n}(D)$ with $\tau(g)=g$. Due to this observation it is only a minor restriction if we focus on the standard symplectic involution $\tau$.

1D. Structure of this article. In Section 2 we give a short general treatment of smooth group schemes over Dedekind rings which are associated with orders in central simple algebras. In particular we treat integral models of inner forms of the special linear group. Further, we consider the fixed points groups attached to involutions. An important tool in the proof of the main theorem will be the pfaffian as a map in nonabelian Galois cohomology (Section 2D). In Section 3 we give an adelic reformulation of Harder's Gauss-Bonnet theorem which hinges on the notion of smooth group scheme. The calculation of the Lefschetz number is based on Rohlfs' method which we summarise in Section 4. Finally the proof of the main theorem is contained in Section 5. It consists of two major steps. The first is the analysis of various nonabelian Galois cohomology sets which occur in Rohlfs' decomposition. The second step is the calculation of the Euler characteristics of the fixed point groups using Harder's Gauss-Bonnet theorem.

Notation. Apart from Section 2, where we work in a more general setting, we use the following notation: $F$ is an algebraic number field and 0 denotes its ring of integers. Let $V$ denote the set of places of $F$. We have $V=V_{\infty} \cup V_{f}$, where $V_{\infty}$ and $V_{f}$ denote the set of Archimedean and finite places of $F$, respectively. Let $v \in V$ be a place of $F$; we denote the completion of $F$ at $v$ by $F_{v}$. The valuation ring of $F_{v}$ is denoted by $\mathrm{O}_{v}$ and the prime ideal in $\mathrm{O}_{v}$ is denoted by $\mathfrak{p}_{v}$. For a nonzero ideal $\mathfrak{a} \subseteq \mathcal{O}$ the ideal norm is defined by $\mathrm{N}(\mathfrak{a}):=|\mathcal{O} / \mathfrak{a}|$. As usual $\mathbb{A}$ denotes the ring of adeles of $F$ and $\mathbb{A}_{f}$ is the ring of finite adeles.

\section{Group schemes associated with orders in central simple algebras}

In this section we will investigate the smoothness properties of group schemes attached to orders in central simple algebras. Throughout, $R$ denotes a Dedekind ring and $k$ denotes its field of fractions. For simplicity we assume $\operatorname{char}(k)=0$. In our applications $R$ is usually the ring of integers of an algebraic number field or a complete discrete valuation ring.

The term scheme always refers to an affine scheme of finite type; the same holds for group schemes. Recall that a scheme $\mathfrak{X}$ defined over $R$ is smooth if for every commutative $R$-algebra $C$ and every nilpotent ideal $I \subseteq C$ the induced map $\mathfrak{X}(C) \rightarrow \mathfrak{X}(C / I)$ is surjective. Suppose $R$ is a complete discrete valuation ring and let $\mathfrak{p}$ denote its prime ideal. We will frequently use the following property: if $\mathfrak{X}$ is a smooth $R$-scheme, then the induced map $\mathfrak{X}(R) \rightarrow \mathfrak{X}\left(R / \mathfrak{p}^{e}\right)$ is surjective 
for every integer $e \geq 1$ [Grothendieck 1964, Corollary 19.3.11]. If $G$ is a group scheme, then we denote the Lie algebra of $G$ by $\operatorname{Lie}(G)$.

2A. The general linear group over an order. Let $A$ be a central simple $k$-algebra and let $\Lambda$ be an $R$-order in $A$. Since $\Lambda$ is a finitely generated torsion-free $R$-module, it is a finitely generated projective $R$-module [Reiner 2003, Theorem (4.13)]. The functor $\Lambda_{a}$ from the category of commutative $R$-algebras to the category of rings defined by $C \mapsto \Lambda \otimes_{R} C$ is represented by the symmetric algebra $S_{R}\left(\Lambda^{*}\right)$, where $\Lambda^{*}=\operatorname{Hom}_{R}(\Lambda, R)$. In fact it defines a smooth $R$-scheme [Grothendieck 1964, Proposition 19.3.2].

Recall that, since $\Lambda$ is finitely generated and projective, one can attach to every $R$-linear endomorphism $\varphi$ of $\Lambda$ its determinant $\operatorname{det}(\varphi) \in R$. More precisely, here the determinant of $\varphi$ is just the determinant of the $k$-linear extension $\varphi \otimes \operatorname{Id}_{k}: A \rightarrow A$. As usual one defines the norm of an element $x \in \Lambda$ to be the determinant of the left multiplication with $x$. One can check that the norm defines a morphism of schemes over $R$

$$
\mathrm{N}_{\Lambda / R}: \Lambda_{a} \rightarrow \mathbb{A}^{1} / R
$$

to the affine line $\mathbb{A}^{1}$ defined over $R$. This can be seen, for instance, by observing that the norm is a natural transformation of functors. Let $C$ be a commutative $R$-algebra. An element $x \in \Lambda \otimes_{R} C$ is a unit if and only if $\mathrm{N}_{\Lambda / R}(x) \in C^{\times}$. It follows from the next lemma that the associated unit group functor $\mathrm{GL}_{\Lambda}: C \mapsto\left(\Lambda \otimes_{R} C\right)^{\times}$is a smooth group scheme over $R$.

Lemma 2.1. Let $\mathbb{A}^{1}$ denote the affine line over $R$. Let $\mathfrak{X}$ be an affine scheme over $R$ with a morphism $f: \mathfrak{X} \rightarrow \mathbb{A}^{1}$. The subfunctor $\mathfrak{Y}$ (from the category of commutative $R$-algebras to the category of sets) defined by

$$
C \mapsto\left\{y \in \mathfrak{X}(C) \mid f(y) \in C^{\times}\right\}
$$

is an affine scheme and the natural transformation $\mathfrak{Y} \rightarrow \mathfrak{X}$ is a morphism of schemes. If $\mathfrak{X}$ is smooth, then $\mathfrak{Y}$ has the same property.

Proof. Let $R[\mathfrak{X}]$ be the coordinate ring of $\mathfrak{X}$ and let $P \in R[\mathfrak{X}]$ be the polynomial defining $f$. Note that $\mathfrak{Y}$ is canonically isomorphic to the functor given by

$$
C \mapsto\{(y, z) \in \mathfrak{X}(C) \times C \mid f(y) z=1\} .
$$

Using this it is easily checked that the $R$-algebra $S:=R[\mathfrak{X}] \otimes_{R} R[T] /(P \otimes T-1)$ represents $\mathfrak{Y}$. Clearly, $S$ is of finite type since $R[\mathfrak{X}]$ is of finite type.

It remains to show that $\mathfrak{Y}$ is smooth whenever $\mathfrak{X}$ is smooth. Assume that $\mathfrak{X}$ is smooth and take a commutative $R$-algebra $C$ with an ideal $J$ such that $J^{2}=0$. By assumption $\mathfrak{X}(C) \rightarrow \mathfrak{X}(C / J)$ is surjective, so given $y \in \mathfrak{Y}(C / J)$ we find $x \in \mathfrak{X}(C)$ projecting to $y$. By assumption $f(x)+J$ is a unit in $C / J$. In particular, 
we can find $z \in C$ with $f(x) z \in 1+J$. However, $1+J$ consists entirely of units and thus $f(x) \in C^{\times}$. We deduce that $\mathfrak{Y}$ is smooth.

We stress this once more: in this article $\mathrm{GL}_{\Lambda}$ is always a functor and not a group. If we take $\Lambda=R$ then we call $\mathrm{GL}_{\Lambda}$ the multiplicative group (or multiplicative group scheme) defined over $R$, and we denote it by $\mathbb{G}_{m}$. Note that the norm defines a homomorphism of $R$-group schemes

$$
\mathrm{N}_{\Lambda / R}: \mathrm{GL}_{\Lambda} \rightarrow \mathbb{G}_{m}
$$

We also point out that the Lie algebra of $\mathrm{GL}_{\Lambda}$ can be (and will be) identified with $\Lambda_{a}$ in a natural way.

\section{B. The special linear group over an order.}

2B1. Reduced norm and trace. Let $A$ be a central simple $k$-algebra. We consider the reduced norm and trace (for definitions see [Reiner 2003, Section 9] or [Weil 1995, Chapter IX, §2]). It was observed by Weil that the reduced norm and trace are polynomial functions. We reformulate this in schematic language: there is a unique element $\operatorname{nrd}_{A / k}$ in the symmetric algebra $S_{k}\left(A^{*}\right)$ (here $A^{*}=\operatorname{Hom}_{k}(A, k)$ ) such that for every splitting field $\ell$ of $A$ and every splitting $\varphi: A \otimes_{k} \ell \stackrel{\simeq}{\longrightarrow} M_{n}(\ell)$ the induced map

$$
S\left(\varphi^{*}\right): S_{\ell}\left(M_{n}(\ell)^{*}\right) \rightarrow S_{k}\left(A^{*}\right) \otimes_{k} \ell
$$

maps the determinant to $\operatorname{nrd}_{A / k} \otimes 1$. Similarly there is the reduced $\operatorname{trace} \operatorname{trd}_{A / k} \in A^{*}$ with an analogous property.

Let $\Lambda \subseteq A$ be an $R$-order. We show that the reduced norm and trace are defined over $R$ in the appropriate sense. For the reduced trace this is easy: elements in $\Lambda$ are integral over $R$, hence the reduced trace takes values in $R$ on the order $\Lambda$ and defines an $R$-linear map $\Lambda \rightarrow R$. In particular we obtain a morphism of schemes over $R$ :

$$
\operatorname{trd}_{\Lambda / R}: \Lambda_{a} \rightarrow \mathbb{A}^{1} / R
$$

Consider the reduced norm. From [Reiner 2003, (9.7)] one can deduce that $\operatorname{nrd}_{A / k}^{n}$ and $\mathrm{N}_{\Lambda / R}$ agree as elements in the coordinate ring $S_{k}\left(A^{*}\right)$. However, the coordinate ring $S_{R}\left(\Lambda^{*}\right)$ of $\Lambda_{a}$ is integrally closed in $S_{k}\left(A^{*}\right)$ and we conclude that the reduced norm is defined over $R$. This means that there is a polynomial $\operatorname{nrd}_{\Lambda / R} \in S_{R}\left(\Lambda^{*}\right)$ that defines the reduced norm as a morphism of $R$-schemes:

$$
\operatorname{nrd}_{\Lambda / R}: \Lambda_{a} \rightarrow \mathbb{A}^{1}
$$

We can also restrict the reduced norm to the unit group and obtain a homomorphism of group schemes:

$$
\operatorname{nrd}_{\Lambda / R}: \mathrm{GL}_{\Lambda} \rightarrow \mathbb{G}_{m} / R
$$


Definition. The special linear group $\mathrm{SL}_{\Lambda}$ over the order $\Lambda$ is the group scheme over $R$ defined by the kernel of the reduced norm:

$$
\mathrm{SL}_{\Lambda}=\operatorname{ker}\left(\operatorname{nrd}_{\Lambda / R}: \mathrm{GL}_{\Lambda} \rightarrow \mathbb{G}_{m}\right) .
$$

2B2. Smoothness of the special linear group. Whereas the general linear group is always smooth, independent of the chosen order, the smoothness of the special linear group depends on the underlying order. Recall the following useful result.

Proposition 2.2 (smoothness of kernels). Let $f: G \rightarrow H$ be a morphism between two smooth group schemes over $R$. If the derivative $\mathrm{d}(f): \operatorname{Lie}(G)(R) \rightarrow$ $\operatorname{Lie}(H)(R)$ is surjective, then the group scheme $K:=\operatorname{ker}(f)$ is smooth over $R$.

Proof. This follows from the theorem of infinitesimal points (see [Demazure and Gabriel 1970, p. 208]) and some easy diagram chasing.

As a matter of fact the derivative of the reduced norm $\mathrm{d}\left(\operatorname{nrd}_{\Lambda / R}\right): \Lambda_{a} \rightarrow \mathbb{A}^{1}$ is the reduced trace. Having this in mind we make the following definition.

Definition. An $R$-order $\Lambda$ in a central simple $k$-algebra is called smooth if the reduced trace $\operatorname{trd}_{\Lambda / R}: \Lambda \rightarrow R$ is surjective.

Note that smoothness of orders is a local property.

Corollary 2.3. If the order $\Lambda$ is smooth then the scheme $\mathrm{SL}_{\Lambda}$ is smooth.

Proof. This follows immediately from Proposition 2.2 using the fact that the derivative of the reduced norm is the reduced trace.

In fact, the converse statement also holds under the assumption $\operatorname{char}(R)=0$. However, we shall not need this result. The next proposition shows that smooth orders exist.

Proposition 2.4. Assume that $R / \mathfrak{p}$ is finite for every prime ideal $\mathfrak{p}$. Then every maximal $R$-order in a central simple $k$-algebra is smooth.

Proof. Let $A$ be a central simple $k$-algebra and let $\Lambda \subset A$ be a maximal $R$-order. Since $\Lambda$ is maximal in $A$ if and only if all $\mathfrak{p}$-adic completions are maximal orders [Reiner 2003, Corollary (11.6)], and since smoothness of $\Lambda$ is a local property, we may assume that $R$ is a complete discrete valuation ring. Recall that $A$ is isomorphic to a matrix algebra $M_{r}(D)$ over a central division algebra $D$. Moreover, $D$ has a unique maximal $R$-order $\Delta \subseteq D$ and $\Lambda$ is (up to conjugation) the maximal order $M_{r}(\Delta)$ in $A$ [Reiner 2003, Theorem (17.3)]. It is known that the reduced trace of a matrix $x=\left(x_{i j}\right)_{i, j=1}^{r} \in M_{r}(D)$ is given by

$$
\operatorname{trd}_{A / k}(x)=\sum_{i=1}^{r} \operatorname{trd}_{D / k}\left(x_{i i}\right)
$$


[Weil 1995, Corollary 2, Chapter IX, §2]. Hence we may assume that $A=D$ is a division algebra and $\Lambda=\Delta$ is the unique maximal order. Let $\operatorname{dim}_{k} D=n^{2}$ and let $\ell / k$ be the unique unramified extension of $k$ of degree $[\ell: k]=n$. The field $\ell$ embeds into $D$ as a maximal subfield and the reduced $\operatorname{trace} \operatorname{trd}_{D / k}$ on the elements of $\ell$ agrees with the field trace $\operatorname{Tr}_{\ell / k}$ [Reiner 2003, proof of Theorem (14.9)]. Let $o_{\ell}$ denote the valuation ring of $\ell$. The image of $o_{\ell}$ under the embedding $\ell \rightarrow D$ lies in the maximal order $\Delta$. Finally the surjectivity of $\operatorname{trd}_{D / k}: \Delta \rightarrow R$ follows from the well-known surjectivity of the field trace $\operatorname{Tr}_{\ell / k}: o_{\ell} \rightarrow R$.

2C. Involutions and fixed point groups. Let $A$ be a central simple $k$-algebra. An involution $\tau$ on $A$ is an additive mapping $\tau: A \rightarrow A$ of order two such that $\tau(x y)=\tau(y) \tau(x)$ for all $x, y \in A$. We say that $\tau$ is of the first kind if $\tau$ is $k$-linear. Otherwise, we say that $\tau$ is of the second kind. In this article all involutions are of the first kind unless the contrary is explicitly stated. We will mostly focus on involutions of symplectic type.

Definition. We say that an involution $\tau$ on $A$ is of symplectic type if there is a splitting field $\ell$ of the algebra $A$, a splitting

$$
\varphi: A \otimes_{k} \ell \stackrel{\simeq}{\longrightarrow} M_{2 n}(\ell),
$$

and a skew-symmetric matrix $a \in M_{2 n}(\ell)$ satisfying $\varphi(\tau(x))=a \varphi(x)^{T} a^{-1}$ for all elements $x \in A \otimes_{k} \ell$. If this is the case, then every splitting (over any splitting field) admits such a matrix.

Let $\tau: A \rightarrow A$ be an involution of the first kind. Let $\Lambda$ be an $R$-order in $A$ and assume that $\Lambda$ is $\tau$-stable. Since $\tau: \Lambda \rightarrow \Lambda$ is $R$-linear, we obtain a morphism of $R$-schemes

$$
\tau: \Lambda_{a} \rightarrow \Lambda_{a} .
$$

We restrict $\tau$ to the unit group $\mathrm{GL}_{\Lambda}$ and compose it with the group inversion to obtain a homomorphism of group schemes

$$
\tau^{*}: \mathrm{GL}_{\Lambda} \rightarrow \mathrm{GL}_{\Lambda} .
$$

We define $G(\Lambda, \tau)$ to be the group of fixed points of $\tau^{*}$, that is, for every commutative $R$-algebra $C$ we obtain

$$
G(\Lambda, \tau)(C)=\left\{x \in\left(\Lambda \otimes_{R} C\right)^{\times} \mid \tau(x) x=1\right\} .
$$

We analyse the smoothness properties of group schemes constructed in this way. Define the $R$-submodule $\operatorname{Sym}(\Lambda, \tau)=\{x \in \Lambda \mid \tau(x)=x\}$ of $\Lambda$ and note that it is a direct summand.

Lemma 2.5. For every commutative $R$-algebra $C$, every $y \in \Lambda \otimes_{R} C$ and every $x \in \operatorname{Sym}(\Lambda, \tau) \otimes_{R} C$ we have $\tau(y) x y \in \operatorname{Sym}(\Lambda, \tau) \otimes_{R} C$. 
Proof. We can write $y=\sum_{i} u_{i} \otimes c_{i}$ for certain $u_{i} \in \Lambda$ and $c_{i} \in C$. The claim is linear in $x$, hence we may assume $x=e \otimes c$ with $e \in \operatorname{Sym}(\Lambda, \tau)$ and $c \in C$. We calculate

$$
\begin{aligned}
\tau(y) x y & =\sum_{i, j} \tau\left(u_{i}\right) e u_{j} \otimes c c_{i} c_{j} \\
& =\sum_{i} \tau\left(u_{i}\right) e u_{i} \otimes c c_{i}^{2}+\sum_{i<j}\left(\tau\left(u_{i}\right) e u_{j}+\tau\left(u_{j}\right) e u_{i}\right) \otimes c c_{i} c_{j},
\end{aligned}
$$

and see that $\tau(y) x y \in \operatorname{Sym}(\Lambda, \tau) \otimes_{R} C$ since $\tau\left(u_{i}\right) e u_{i}$ and $\tau\left(u_{i}\right) e u_{j}+\tau\left(u_{j}\right) e u_{i}$ are elements of $\operatorname{Sym}(\Lambda, \tau)$.

Definition. The order $\Lambda$ is called $\tau$-smooth if the map $s: \Lambda \rightarrow \operatorname{Sym}(\Lambda, \tau)$ defined by $x \mapsto x+\tau(x)$ is surjective. Clearly $\tau$-smoothness is a local property.

Proposition 2.6. If an $R$-order $\Lambda$ is $\tau$-smooth, then the scheme $G(\Lambda, \tau)$ is smooth.

Proof. We set $G:=G(\Lambda, \tau)$. Let $C$ be a commutative $R$-algebra with an ideal $I \subseteq C$ such that $I^{2}=0$. We have to show that the canonical map $G(C) \rightarrow G(C / I)$ is surjective. Take $\bar{y} \in G(C / I)$. Since the unit group scheme $\mathrm{GL}_{\Lambda}$ is smooth (see Section 2A), we can find $y \in \mathrm{GL}_{\Lambda}(C)=(\Lambda \otimes C)^{\times}$mapping to $\bar{y}$ modulo $I$. Since $\bar{y}$ is in the fixed point group of $\tau^{*}$, this implies that

$$
\tau(y) y=1+\rho
$$

with some $\rho \in \Lambda \otimes I$.

We consider $E:=\operatorname{Sym}(\Lambda, \tau)$ and we obtain $\tau(y) y \in E \otimes_{R} C$ by Lemma 2.5. Consequently, there is $u \in \Lambda \otimes_{R} C$ such that $\tau(u)+u=y$. Moreover, $1 \in E$; thus there is some $v \in \Lambda \otimes_{R} C$ with $\tau(v)+v=1$. We deduce that $\rho=\tau(u-v)+(u-v)$ is an element in $E \otimes_{R} C$, and thus

$$
\rho \in\left(E \otimes_{R} C\right) \cap\left(\Lambda \otimes_{R} I\right)=E \otimes_{R} I .
$$

As a last step we use once again that $\Lambda$ is $\tau$-smooth and deduce that there is some $w \in \Lambda \otimes I$ with $\rho=\tau(w)+w$. We put $y^{\prime}:=y(1-w)$, which is congruent to $\bar{y}$ modulo $I$ and satisfies

$$
\begin{aligned}
\tau\left(y^{\prime}\right) y^{\prime} & =(1-\tau(w)) \tau(y) y(1-w)=(1-\tau(w))(1+\rho)(1-w) \\
& =1+\rho-\tau(w)-w=1 .
\end{aligned}
$$

Therefore $y^{\prime} \in G(C)$ and $y^{\prime}$ maps to $\bar{y} \in G(C / I)$ under the canonical map.

2D. Involutions of symplectic type and the pfaffian. Let $A$ be a central simple $k$-algebra with an involution of symplectic type $\tau$. Let $\Lambda$ be a $\tau$-stable $R$-order in $A$. 
2D1. The pfaffian. Set $E:=\operatorname{Sym}(\Lambda, \tau)$ in the notation of Section $2 C$. The inclusion $\iota: E \rightarrow \Lambda$ induces a morphism of $R$-algebras

$$
S\left(\iota^{*}\right): S_{R}\left(\Lambda^{*}\right) \rightarrow S_{R}\left(E^{*}\right) .
$$

Recall that the reduced norm is given by a polynomial function $\operatorname{nrd}_{\Lambda / R} \in S_{R}\left(\Lambda^{*}\right)$ (see Section 2B1). We define $\operatorname{nrd}_{\mid E}:=S\left(\iota^{*}\right)\left(\operatorname{nrd}_{\Lambda / R}\right) \in S_{R}\left(E^{*}\right)$. We will construct a pfaffian, that is, a polynomial $\mathrm{pf}_{\tau} \in S_{R}\left(E^{*}\right)$ such that $\operatorname{nrd}_{\mid E}=\mathrm{pf}_{\tau}^{2}$.

Let $L / k$ be any field extension. It follows from [Knus et al. 1998, Proposition 2.9] that for every $x \in E \otimes_{R} L$ the reduced norm $\operatorname{nrd}_{\mid E}(x)$ is a square in $L$. Therefore, we may deduce that there is a polynomial $f \in S_{R}\left(E^{*}\right)$ such that

$$
f^{2}=\operatorname{nrd}_{\mid E} .
$$

We normalise this polynomial $\mathrm{pf}_{\tau}:= \pm f$ such that $\mathrm{pf}_{\tau}(1)=1$ and we call $\mathrm{pf}_{\tau}$ the pfaffian with respect to $\tau$.

Lemma 2.7. Let $S\left(\tau^{*}\right)$ denote the automorphism of the symmetric $R$-algebra $S_{R}\left(\Lambda^{*}\right)$ which is induced by $\tau$. The following assertions hold:

(i) $S\left(\tau^{*}\right)\left(\operatorname{nrd}_{\Lambda / R}\right)=\operatorname{nrd}_{\Lambda / R}$.

(ii) For all $y \in \Lambda \otimes_{R} C$ and all $x \in \operatorname{Sym}(\Lambda, \tau) \otimes_{R} C$, we have

$$
\operatorname{pf}_{\tau}(\tau(y) x y)=\operatorname{nrd}_{\Lambda / R}(y) \operatorname{pf}_{\tau}(x),
$$

where $C$ is any commutative $R$-algebra.

Proof. To prove the first claim we may work over fields. However, over fields this is the well-known statement [Knus et al. 1998, Corollary 2.2].

The same proof works for the second statement. Note that $\tau(y) x y$ lies in $\operatorname{Sym}(\Lambda, \tau) \otimes_{R} C$ by Lemma 2.5. Both are polynomial functions on $\Lambda \times \operatorname{Sym}(\Lambda, \tau)$. If they agree over all fields then they agree as polynomials. However, over fields this is the result [Knus et al. 1998, Proposition 2.13].

Remark. Consider the fixed point group scheme $G=G(\Lambda, \tau)$ associated with $\tau$. Let $x \in G(C)$ for some commutative $R$-algebra $C$. We see from $\tau(x) x=1$ and Lemma 2.7 that

$$
\operatorname{nrd}_{\Lambda / R}(x)=\operatorname{pf}_{\tau}(\tau(x) x)=\operatorname{pf}_{\tau}(1)=1 .
$$

Hence the reduced norm restricts to the trivial character on $G(\Lambda, \tau)$.

2D2. The cohomological pfaffian. We study nonabelian Galois cohomology of $\tau^{*}$ with values in the groups $\mathrm{GL}_{\Lambda}(C)$ and $\mathrm{SL}_{\Lambda}(C)$. For the definition of nonabelian cohomology we refer the reader to [Serre 1994; 1979, pages 123-126] or [Knus et al. 1998, Chapter VII]. We shall in this context often denote $\tau$ and $\tau^{*}$ by left exponents, that is, we write $\tau_{x}^{*}$ for $\tau^{*}(x)$. 
Let $C$ be a commutative $R$-algebra and assume that $C$ is flat as an $R$-module. A cocycle $b$ in $Z^{1}\left(\tau^{*}, \mathrm{GL}_{\Lambda}(C)\right)$ is an element of $(\Lambda \otimes C)^{\times}$which satisfies $b^{\tau^{*}} b=1$, or equivalently $b={ }^{\tau} b$. In other words

$$
Z^{1}\left(\tau^{*}, \mathrm{GL}_{\Lambda}(C)\right)=\operatorname{Sym}\left(\Lambda \otimes_{R} C, \tau\right) \cap \mathrm{GL}_{\Lambda}(C) .
$$

The assumption that $C$ is flat yields that $\operatorname{Sym}\left(\Lambda \otimes_{R} C, \tau\right)=\operatorname{Sym}(\Lambda, \tau) \otimes_{R} C$. Therefore we can apply the pfaffian associated with $\tau$ to cocycles in $Z^{1}\left(\tau^{*}, \mathrm{GL}_{\Lambda}(C)\right)$. Two cocycles $b$ and $c$ are cohomologous if there is $y \in \mathrm{GL}_{\Lambda}(C)$ such that $b={ }^{\tau} y c y$. In this case it follows from Lemma 2.7 that $\operatorname{pf}_{\tau}(b)=\operatorname{nrd}_{\Lambda / R}(y) \operatorname{pf}_{\tau}(c)$. Therefore the pfaffian defines a morphism of pointed sets

$$
\mathrm{pf}_{\tau}: H^{1}\left(\tau^{*}, \mathrm{GL}_{\Lambda}(C)\right) \rightarrow C^{\times} / \operatorname{nrd}_{\Lambda / R}\left(\mathrm{GL}_{\Lambda}(C)\right) .
$$

By the same reasoning we obtain a morphism of pointed sets

$$
\mathrm{pf}_{\tau}: H^{1}\left(\tau^{*}, \mathrm{SL}_{\Lambda}(C)\right) \rightarrow\left\{x \in C^{\times} \mid x^{2}=1\right\} .
$$

For simplicity we define $C^{(2)}:=\left\{x \in C^{\times} \mid x^{2}=1\right\}$ and $C_{\Lambda}^{\times}:=\operatorname{nrd}_{\Lambda / R}\left(\mathrm{GL}_{\Lambda}(C)\right)$.

Proposition 2.8 (cohomological diagram for symplectic involutions). Let $\tau$ be an involution of symplectic type on $A$ and let $\Lambda$ be a $\tau$-stable $R$-order. For every commutative $R$-algebra $C$ which is flat as an $R$-module, there is a commutative diagram of pointed sets with exact rows:

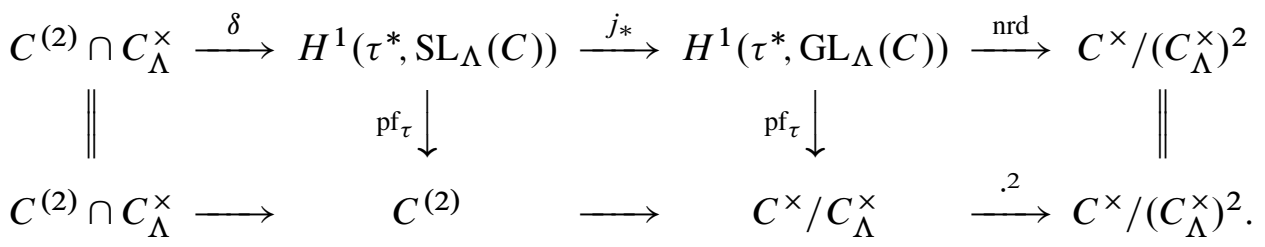

The map $\delta$ is injective and the lower row is an exact sequence of groups. Here $j_{*}$ denotes the map induced by the inclusion $j: \mathrm{SL}_{\Lambda}(C) \rightarrow \mathrm{GL}_{\Lambda}(C)$.

Proof. The short exact sequence of groups

$$
1 \longrightarrow \mathrm{SL}_{\Lambda}(C) \stackrel{j}{\longrightarrow} \mathrm{GL}_{\Lambda}(C) \stackrel{\mathrm{nrd}}{\longrightarrow} C_{\Lambda}^{\times} \longrightarrow 1
$$

is an exact sequence of groups with $\tau^{*}$-action, where $\tau^{*}$ acts on $C_{\Lambda}^{\times}$by inversion. Consider the initial segment of the associated long exact sequence in the cohomology (see [Serre 1994, Proposition I.38]):

$$
1 \longrightarrow \mathrm{SL}_{\Lambda}(C)^{\tau^{*}} \stackrel{j}{\longrightarrow} \mathrm{GL}_{\Lambda}(C)^{\tau^{*}} \stackrel{\mathrm{nrd}}{\longrightarrow} C_{\Lambda}^{\times} \cap C^{(2)} \longrightarrow \cdots .
$$


It follows from the remark on page 379 that $\mathrm{SL}_{\Lambda}(C)^{\tau^{*}} \stackrel{j}{\longrightarrow} \mathrm{GL}_{\Lambda}(C)^{\tau^{*}}$ is bijective. Thus the long exact sequence takes the form

$$
1 \longrightarrow C_{\Lambda}^{\times} \cap C^{(2)} \stackrel{\delta}{\longrightarrow} H^{1}\left(\tau^{*}, \mathrm{SL}_{\Lambda}(C)\right) \longrightarrow H^{1}\left(\tau^{*}, \mathrm{GL}_{\Lambda}(C)\right) \longrightarrow H^{1}\left(\tau^{*}, C_{\Lambda}^{\times}\right) .
$$

It is easy to see that $H^{1}\left(\tau^{*}, C_{\Lambda}^{\times}\right)=C_{\Lambda}^{\times} /\left(C_{\Lambda}^{\times}\right)^{2}$, which is a subgroup of $C^{\times} /\left(C_{\Lambda}^{\times}\right)^{2}$. Hence we simply replace the last term by $C^{\times} /\left(C_{\Lambda}^{\times}\right)^{2}$. This yields the upper row of the diagram. It is an easy exercise to verify that the lower row is an exact sequence of groups.

It remains to verify the commutativity of the rectangles. The middle one is obviously commutative by definition of the pfaffian in the cohomology. For the last rectangle we simply use that $\operatorname{pf}_{\tau}(g)^{2}=\operatorname{nrd}(g)$ for all $g \in Z^{1}\left(\tau^{*}, \mathrm{GL}_{\Lambda}(C)\right)$ by the construction of the pfaffian.

Consider the first rectangle. We recall the definition of the connecting morphism $\delta$ : given $c \in C_{\Lambda}^{\times} \cap C^{(2)}$, we can find an element $g \in \mathrm{GL}_{\Lambda}(C)$ such that $\operatorname{nrd}_{\Lambda / R}(g)=c$; then $\delta(c)$ is defined to be the class of $g^{-1} \tau_{g}^{*}$. The pfaffian of $g^{-1} \tau_{g}^{*}$ is

$$
\operatorname{pf}_{\tau}\left(g^{-1} \tau_{g}^{*}\right)=\operatorname{nrd}(g)^{-1}=c^{-1}=c
$$

(see Lemma 2.7). This proves the commutativity of the first rectangle.

Finally, note that $\delta$ is injective since $\mathrm{pf}_{\tau} \circ \delta$ is injective.

Corollary 2.9. An element $x \in H^{1}\left(\tau^{*}, \mathrm{GL}_{\Lambda}(C)\right)$ lies in the image of $j_{*}$ if and only if $\mathrm{pf}_{\tau}(x)$ lies in the image of the canonical map $C^{(2)} \rightarrow C^{\times} / C_{\Lambda}^{\times}$.

Proof. Let $\alpha: C^{(2)} \rightarrow C^{\times} / C_{\Lambda}^{\times}$denote the canonical map. Suppose the class $x \in H^{1}\left(\tau^{*}, \mathrm{GL}_{\Lambda}(C)\right)$ is in the image of $j_{*}$, then we obtain immediately that $\mathrm{pf}_{\tau}(x)$ lies in the image of $\alpha$.

Conversely, suppose $\operatorname{pf}_{\tau}(x)=\alpha(u)$ for some $u \in C^{(2)}$. Then the diagram shows that $\operatorname{nrd}_{\Lambda / R}(x)$ is 1 in $C^{\times} /\left(C_{\Lambda}^{\times}\right)^{2}$ and therefore $x$ lies in the image of $j_{*}$.

Remark (twisting involutions). Let $A$ be a central simple $k$-algebra with an involution $\tau$ of symplectic type and let $\Lambda$ be a $\tau$-stable $R$-order. Given an element $b \in \operatorname{Sym}(\Lambda, \tau) \cap \Lambda^{\times}$, we can twist the involution $\tau$ with $b$. More precisely, we define $\tau \mid b: A \rightarrow A$ by $x \mapsto b^{\tau} x b^{-1}$. It is easily verified that this is again an involution on $A$, and since $b \in \Lambda^{\times}$, the order $\Lambda$ is $\tau \mid b$-stable. Note that $\tau \mid b$ is again an involution of symplectic type.

Suppose $\Lambda$ is $\tau$-smooth, we claim that $\Lambda$ is $\tau \mid b$-smooth as well. Take some element $y$ in $\operatorname{Sym}(\Lambda, \tau \mid b)$; this is $y=b^{\tau} y b^{-1}$. Consequently, $y b \in \operatorname{Sym}(\Lambda, \tau)$ and by $\tau$-smoothness there is an element $z \in \Lambda$ which satisfies ${ }^{\tau} z+z=y b$. The element $b$ is a unit in $\Lambda$, hence we may write $z=w b$ for $w=z b^{-1} \in \Lambda$ and it follows that $\tau \mid b w+w=y$. We have shown that $\Lambda$ is $\tau \mid b$-smooth.

Finally, for all $b \in \operatorname{Sym}(\Lambda, \tau) \cap \Lambda^{\times}$we have $(\tau \mid b)^{*}=\operatorname{int}(b) \circ \tau^{*}$ on the group scheme $\mathrm{GL}_{\Lambda}$. Since $b={ }^{\tau} b$ is equivalent to $b^{\tau^{*}} b=1$, such an element $b$ is a 
cocycle for $H^{1}\left(\tau^{*}, \Lambda^{\times}\right)$. If we now twist $\tau^{*}$ with the cocycle $b$ (see Section 4 ), we obtain

$$
\tau^{*} \mid b:=\operatorname{int}(b) \circ \tau^{*}=(\tau \mid b)^{*} .
$$

2E. Hermitian forms and nonabelian Galois cohomology. We shall also need a result due to Fainsilber and Morales from the theory of hermitian forms. Let $A$ be a central simple $k$-algebra and let $\tau$ be an involution on $A$. In this short section it is not important whether or not $\tau$ is of the first or of the second kind.

The notion of $\tau$-smoothness is related to the theory of even hermitian forms. Let $\Lambda$ be a $\tau$-stable $R$-order in $A$ and let $M$ be a finitely generated and projective right $\Lambda$-module. A hermitian form $h$ (or more precisely a 1-hermitian form) with respect to $\tau$ on $M$ is said to be even if there is a $\tau$-sesquilinear form $s: M \times M \rightarrow \Lambda$ such that $h=s+s^{*}$. Here $s^{*}$ is the sesquilinear form defined by

$$
s^{*}(x, y):={ }_{s}(y, x) .
$$

It follows immediately that $\Lambda$ is $\tau$-smooth if and only if every hermitian form on $\Lambda$ (considered as a right $\Lambda$-module) is even. This is useful since even hermitian forms can be handled more easily than arbitrary hermitian forms.

We consider the automorphism $\tau^{*}$ of $\Lambda^{\times}$defined as the composition of $\tau$ and the group inversion. Similarly we obtain $\tau^{*}$ on $A^{\times}$. Here it is not necessary to consider $\tau^{*}$ as a morphism of group schemes, which is a little bit more tedious if $\tau$ is of the second kind. We will need a theorem from [Fainsilber and Morales 1999] in the following paraphrase:

Theorem 2.10. Let $k$ be a field which is complete for a discrete valuation and let $R$ be its valuation ring. Let $A$ be a central simple $k$-algebra with involution $\tau$. Suppose $\Lambda$ is a $\tau$-stable maximal $R$-order in $A$. If $\Lambda$ is $\tau$-smooth, the canonical map

$$
j_{*}: H^{1}\left(\tau^{*}, \Lambda^{\times}\right) \rightarrow H^{1}\left(\tau^{*}, A^{\times}\right)
$$

is injective.

Compared with [Fainsilber and Morales 1999] we have added the assumption of $\tau$-smoothness to eliminate the restriction on the residual characteristic. The proof is almost identical.

\section{An adelic reformulation of Harder's Gauss-Bonnet theorem}

We briefly describe an adelic reformulation of Harder's Gauss-Bonnet theorem [Harder 1971] that hinges on the notion of a smooth group scheme. In fact, the Euler characteristic of an arithmetic group can also be computed using G. Prasad's [1989] general volume formula. Since we have explicit underlying smooth integral models of the algebraic groups, we think that the adelic volume formula derived in this section is adapted much better to the applications given in this article. 
Let $F$ be an algebraic number field and let $O$ denote its ring of integers. Let $G$ be a connected semisimple algebraic group defined over $F$. We denote by $G_{\infty}$ the associated real semisimple Lie group

$$
G_{\infty}=G\left(F \otimes_{\mathbb{Q}} \mathbb{R}\right)=\prod_{v \in V_{\infty}} G\left(F_{v}\right) .
$$

3A. The Euler-Poincaré measure. We define what we mean by the compact dual group of $G_{\infty}$, since the definition differs from author to author. Let $\mathfrak{g}_{\infty}$ be the real Lie algebra of $G_{\infty}$ and let $\mathfrak{g}_{\infty, \mathbb{C}}$ denote its complexification. Moreover, let $K_{\infty}$ be a maximal compact subgroup of $G_{\infty}$ and consider the associated Cartan decomposition

$$
\mathfrak{g}_{\infty}=\mathfrak{k}_{\infty} \oplus \mathfrak{p}
$$

The real vector space $\mathfrak{u}:=\mathfrak{k}_{\infty} \oplus i \mathfrak{p} \subseteq \mathfrak{g}_{\infty, \mathbb{C}}$ is a real Lie subalgebra of $\mathfrak{g}_{\infty, \mathbb{C}}$ and is even a compact real form of $\mathfrak{g}_{\infty, \mathbb{C}}$ [Knapp 2002, page 360]. Let $G_{u}$ be the unique connected (a priori virtual) Lie subgroup of $G\left(F \otimes_{\mathbb{Q}} \mathbb{C}\right)$ with Lie algebra $\mathfrak{u}$. Since the real semisimple Lie algebra $\mathfrak{u}$ is a compact form, the Lie group $G_{u}$ is compact and thus closed in $G\left(F \otimes_{\mathbb{Q}} \mathbb{C}\right)$ [Knapp 2002, Chapter IV, Theorem 4.69]. Further we see that the connected component $K_{\infty}^{0}$ is a subgroup of $G_{u}$. We say that $G_{u}$ is the compact dual group of $G_{\infty}$ containing $K_{\infty}^{0}$. Note that the dual group depends on the algebraic group $G$.

Let $B: \mathfrak{g}_{\infty} \times \mathfrak{g}_{\infty} \rightarrow \mathbb{R}$ be a nondegenerate $\mathbb{R}$-bilinear form such that $\mathfrak{k}_{\infty}$ and $\mathfrak{p}$ are orthogonal. We extend $B$ to a $\mathbb{C}$-bilinear form (again denoted by $B$ ) on $\mathfrak{g}_{\infty, \mathbb{C}}$. Note that $B$ restricted to $\mathfrak{u}$ is a nondegenerate $\mathbb{R}$-bilinear form $\mathfrak{u} \times \mathfrak{u} \rightarrow \mathbb{R}$. We obtain corresponding right-invariant volume densities on $G_{\infty}$ and on $G_{u}$ which will be denoted by $\left|\operatorname{vol}_{B}\right|$.

We define $X:=K_{\infty} \backslash G_{\infty}$. Let $\Gamma \subseteq G(F)$ be a torsion-free arithmetic group. Harder's Gauss-Bonnet theorem shows that integration over $G_{\infty} / \Gamma$ with the EulerPoincaré measure $\mu_{\chi}$ [Serre 1971, §3] yields the Euler characteristic of $\Gamma$ - even if $\Gamma$ is not cocompact. Via Hirzebruch's proportionality principle one has the following formula for the Euler-Poincaré measure on $G_{\infty}$ [Harder 1971; Serre 1971].

Theorem 3.1. If $\operatorname{dim}(X)$ is odd or if $\operatorname{rk}\left(\mathfrak{k}_{\infty, \mathbb{C}}\right)<\operatorname{rk}\left(\mathfrak{g}_{\infty, \mathbb{C}}\right)$, then $\mu_{\chi}=0$ is the Euler-Poincaré measure. Otherwise, if $\operatorname{rk}\left(\mathfrak{g}_{\infty, \mathbb{C}}\right)=\operatorname{rk}\left(\mathfrak{k}_{\infty, \mathbb{C}}\right)$ and $\operatorname{dim}(X)=2 p$ is even, then

$$
\mu_{\chi}:=\frac{(-1)^{p}\left|W\left(\mathfrak{g}_{\infty, \mathbb{C}}\right)\right|}{\left|\pi_{0}\left(G_{\infty}\right)\right|\left|W\left(\mathfrak{k}_{\infty, \mathbb{C}}\right)\right|} \operatorname{vol}_{B}\left(G_{u}\right)^{-1}\left|\operatorname{vol}_{B}\right|
$$

Here $\pi_{0}\left(G_{\infty}\right)=G_{\infty} / G_{\infty}^{0}$ and $W\left(\mathfrak{g}_{\infty, \mathbb{C}}\right), W\left(\mathfrak{k}_{\infty, \mathbb{C}}\right)$ denote the Weyl groups of the complexified Lie algebras $\mathfrak{g}_{\infty, \mathbb{C}}, \mathfrak{k}_{\infty, \mathbb{C}}$. 
3B. The adelic reformulation. Let $\mathbb{A}$ denote the ring of adeles of $F$ and $\mathbb{A}_{f}$ the ring of finite adeles. Let $G$ be a connected semisimple algebraic group defined over $F$. Let $K_{f} \subseteq G\left(\mathbb{A}_{f}\right)$ be an open compact subgroup of the locally compact group $G\left(\mathbb{A}_{f}\right)$. Borel showed that $G(\mathbb{A})$ is the disjoint union of a finite number $m$ of double cosets, that is,

$$
G(\mathbb{A})=\bigsqcup_{i=1}^{m} G_{\infty} K_{f} x_{i} G(F)
$$

for some representatives $x_{1}, \ldots, x_{m} \in G\left(\mathbb{A}_{f}\right)$ [Borel 1963, Theorem 5.1]. For every $i=1, \ldots, m$ we obtain an arithmetic subgroup $\Gamma_{i} \subseteq G(F)$ defined by

$$
\Gamma_{i}:=G(F) \cap x_{i}^{-1} K_{f} x_{i} .
$$

There is a $G_{\infty}$-equivariant homeomorphism

$$
K_{f} \backslash G(\mathbb{A}) / G(F) \stackrel{\simeq}{\longrightarrow} \bigsqcup_{i=1}^{m} G_{\infty} / \Gamma_{i} .
$$

Here the right-hand side denotes the topologically disjoint union.

Remark. Define $X=K_{\infty} \backslash G_{\infty}$. Suppose $G(F)$ acts freely on $K_{\infty} K_{f} \backslash G(\mathbb{A})$. This is the case if and only if the groups $\Gamma_{i}$ are torsion-free for all $i=1, \ldots, m$. If $\operatorname{dim}(X)$ is odd or if $\operatorname{rk}\left(\mathfrak{k}_{\infty, \mathbb{C}}\right)<\operatorname{rk}\left(\mathfrak{g}_{\infty, \mathbb{C}}\right)$, then

$$
\chi\left(K_{\infty} K_{f} \backslash G(\mathbb{A}) / G(F)\right)=0 .
$$

This follows immediately from Harder's Gauss-Bonnet theorem and the homeomorphism in (1).

Note further that if $F$ has a complex place, then $\operatorname{rk}\left(\mathfrak{k}_{\infty, \mathbb{C}}\right)<\operatorname{rk}\left(\mathfrak{g}_{\infty, \mathbb{C}}\right)$ is always satisfied. Therefore we may restrict to the case where $F$ is totally real.

3B1. The Tamagawa measure. We derive a description of the Tamagawa measure in terms of the local volume densities. For a thorough definition of the Tamagawa measure we refer the reader to [Oesterlé 1984]. Let $G$ be a connected semisimple linear algebraic $F$-group of dimension $d$. Let $\mathfrak{g}=\operatorname{Lie}(G)(F)$ be the Lie algebra of $G$ over $F$.

Fix a nondegenerate $F$-bilinear form $B: \mathfrak{g} \times \mathfrak{g} \rightarrow F$ on the Lie algebra. For every place $v \in V$ we have the left invariant volume density $\left|\operatorname{vol}_{B}\right|_{v}$ attached to $B$ on the $F_{v}$-analytic manifold $G\left(F_{v}\right)$. The volume density is uniquely determined by $\left|\operatorname{vol}_{B}\right|\left(e_{1} \wedge \cdots \wedge e_{d}\right)=\left|\operatorname{det}\left(B\left(e_{i}, e_{j}\right)\right)\right|^{1 / 2}$ for all $e_{1}, \ldots, e_{d} \in \mathfrak{g}$. We fix Haar measures $\mu_{v}$ on $F_{v}$ for every place $v$ such that

(i) $\mu_{v}\left(\mathscr{O}_{v}\right)=1$ if $v \in V_{f}$ is a finite place,

(ii) $\mu_{v}([0,1])=1$ if $v$ is a real place, and

(iii) $\mu_{v}([0,1]+[0,1] i)=2$ if $v$ is a complex place. 
Using these choices of Haar measures, a density on $G\left(F_{v}\right)$ defines a measure on the analytic manifold $G\left(F_{v}\right)$.

Lemma 3.2. Let $G$ be a d-dimensional semisimple connected linear algebraic group defined over $F$. Fix a nondegenerate $F$-bilinear form $B: \mathfrak{g} \times \mathfrak{g} \rightarrow F$ on the Lie algebra. Then the Tamagawa measure on $G(\mathbb{A})$ is given by

$$
\tau=\left|d_{F}\right|^{-d / 2} \prod_{v \in V}\left|\operatorname{vol}_{B}\right|_{v}
$$

Proof. Let $e_{1}, \ldots, e_{d}$ be a basis of $\mathfrak{g}$ over $F$ and take the dual basis $\varepsilon_{1}, \ldots, \varepsilon_{d}$ of $\operatorname{Hom}_{F}(\mathfrak{g}, F)$. We define a nontrivial form of highest degree $\omega=\varepsilon_{1} \wedge \cdots \wedge \varepsilon_{d}$ on $\mathfrak{g}$. By definition of the volume density we have

$$
\left|\operatorname{vol}_{B}\right|_{v}=\left|\operatorname{det}\left(B\left(e_{i}, e_{j}\right)\right)\right|_{v}^{1 / 2}|\omega|_{v}
$$

By the product formula we know that $\left|\operatorname{det}\left(B\left(e_{i}, e_{j}\right)\right)\right|_{v}=1$ for almost all places $v$ and further that $\prod_{v \in V}\left|\operatorname{det}\left(B\left(e_{i}, e_{j}\right)\right)\right|_{v}=1$.

3B2. The modulus factor. We focus on the case where the algebraic group has a smooth 0 -model. Let $G$ be a smooth group scheme defined over $\mathrm{O}$. For any commutative 0 -algebra $R$ we write $\mathfrak{g}_{R}:=\operatorname{Lie}(G)(R)$ to denote the $R$-points of the Lie algebra of $G$. Let $B: \mathfrak{g}_{F} \times \mathfrak{g}_{F} \rightarrow F$ be a nondegenerate $F$-bilinear form. For every finite place $v \in V_{f}$ we define the modulus factor $m(B)_{v}$ as follows: take an $\mathcal{O}_{v}$-basis $e_{1}, \ldots, e_{n}$ of the free $\mathscr{O}_{v}$-module $\mathfrak{g}_{v}:=\mathfrak{g}_{\mathscr{O}_{v}}$, and define

$$
m(B)_{v}:=\left|\operatorname{det}\left(B\left(e_{i}, e_{j}\right)\right)\right|_{v}^{1 / 2}
$$

For almost all finite places $v \in V_{f}$ we have $m(B)_{v}=1$. To see this, take an $F$-basis of $\mathfrak{g}_{F}$ and note that it is an $\mathcal{O}_{v}$-basis of $\mathfrak{g}_{v}$ for almost all finite places $v$. This allows us to define the global modulus factor $m(B):=\prod_{v \in V_{f}} m(B)_{v}$.

3B3. Congruence groups. In the adelic formulation of Harder's Gauss-Bonnet theorem we focus on congruence groups which are given by local data. Let $G$ be a smooth 0 -group scheme. For every finite place $v \in V_{f}$, let $\alpha_{v} \geq 1$ be a natural number and we assume that $\alpha_{v}=1$ for almost all $v \in V_{f}$. Let $v$ be a finite place and let $\mathfrak{p}_{v} \subseteq \mathcal{O}_{v}$ be the unique prime ideal in $\mathcal{O}_{v}$. We define $\pi_{v}$ to be the reduction morphism

$$
\pi_{v}: G\left(\mathscr{O}_{v}\right) \rightarrow G\left(\mathcal{O}_{v} / \mathfrak{p}_{v}^{\alpha_{v}}\right)
$$

Further, we assume that we are given a subgroup $U_{v}$ of the finite group $G\left(\mathscr{O}_{v} / \mathfrak{p}_{v}^{\alpha_{v}}\right)$ for every place $v \in V_{f}$. For a place $v \in V_{f}$ the group $K_{v}(U):=\pi_{v}^{-1}\left(U_{v}\right)$ is an open compact subgroup of $G\left(\mathscr{O}_{v}\right)$. If we additionally impose the assumption that 
$U_{v}=G\left(\mathcal{O}_{v} / \mathfrak{p}_{v}^{\alpha_{v}}\right)$ for almost all $v$, then the group

$$
K(U):=\prod_{v \in V_{f}} K_{v}(U)
$$

is an open compact subgroup of the locally compact group $G\left(\mathbb{A}_{f}\right)$. We say that $K(U)$ is the congruence group associated with the local datum

$$
U=\left(U_{v}\right)_{v}=\left(U_{v}, \alpha_{v}\right)_{v}
$$

(usually the numbers $\alpha_{v}$ are considered to be implicitly a part of the datum $U$ ).

3B4. The adelic Euler characteristic formula. Let $F$ be a totally real number field. Let $G$ be a smooth group scheme over $\mathcal{O}$ such that $G \times_{\mathcal{O}} F$ is a connected semisimple group. For every real place $v$ we choose a maximal compact subgroup $K_{v} \subseteq G\left(F_{v}\right)$. The real Lie algebra of $K_{v}$ will be denoted $\mathfrak{k}_{v}$. The product $K_{\infty}=\prod_{v \in V_{\infty}} K_{v}$ is a maximal compact subgroup of the associated real Lie group $G_{\infty}$. We denote the Lie algebra of $K_{\infty}$ by $\mathfrak{k}$.

Let $B: \mathfrak{g}_{F} \times \mathfrak{g}_{F} \rightarrow F$ be an $F$-bilinear form. We say that $B$ is nice with respect to $K_{\infty}$ if $B$ is nondegenerate and for every real place $v \in V_{\infty}$ the Cartan decomposition with respect to $\mathfrak{k}_{v}$ is orthogonal with respect to $B$. A nice form induces a nondegenerate bilinear form $B: \mathfrak{g}_{\infty} \times \mathfrak{g}_{\infty} \rightarrow \mathbb{R}$ by defining the Lie subalgebras $\mathfrak{g}_{v}=\operatorname{Lie}\left(G\left(F_{v}\right)\right)$ to be orthogonal. Note that the form $B$ satisfies the requirements of Theorem 3.1.

Theorem 3.3. Let $G$ be a smooth group scheme over 0 such that $G \times_{\mathbb{O}} F$ is a connected semisimple group of dimension $d$. We fix any nice form $B: \mathfrak{g}_{F} \times \mathfrak{g}_{F} \rightarrow F$. Furthermore, let $K_{f}=K(U)$ be a congruence subgroup of $G\left(\mathbb{A}_{f}\right)$ given by a local datum $(U, \alpha)$ such that $G(F)$ acts freely on $K_{\infty} K_{f} \backslash G(\mathbb{A})$.

If $\operatorname{dim}(X)=2 p$ is even and $\operatorname{rk}\left(\mathfrak{k}_{\mathbb{C}}\right)=\operatorname{rk}\left(\mathfrak{g}_{\infty}, \mathbb{C}\right)$, then the Euler characteristic of the double coset space $K_{\infty} K_{f} \backslash G(\mathbb{A}) / G(F)$ is given by

$$
\begin{aligned}
& \chi\left(K_{\infty} K_{f} \backslash G(\mathbb{A}) / G(F)\right) \\
&=(-1)^{p}\left|d_{F}\right|^{d / 2} \frac{\left|W\left(\mathfrak{g}_{\infty, \mathbb{C}}\right)\right| \tau(G)}{\left|\pi_{0}\left(G_{\infty}\right)\right|\left|W\left(\mathfrak{k}_{\mathbb{C}}\right)\right|} \operatorname{vol}_{B}\left(G_{u}\right)^{-1} m(B)^{-1} \prod_{v \in V_{f}} \frac{\mathrm{N}\left(\mathfrak{p}_{v}\right)^{d \alpha_{v}}}{\left|U_{v}\right|} .
\end{aligned}
$$

Here $\tau(G)$ is the Tamagawa number of $G, \mathrm{~N}\left(\mathfrak{p}_{v}\right)$ denotes the cardinality of the residue class field $\mathscr{O}_{v} / \mathfrak{p}_{v}$, and $G_{u}$ denotes the compact dual group of $G_{\infty}^{0}$ (remaining notation is as in Theorem 3.1).

Proof. Let $x_{1}, \ldots, x_{m} \in G\left(\mathbb{A}_{f}\right)$ be a collection of representatives of the finitely many elements of $G_{\infty} K_{f} \backslash G(\mathbb{A}) / G(F)$. We consider the torsion-free arithmetic groups $\Gamma_{i}$ defined as $\Gamma_{i}:=G(F) \cap x_{i}^{-1} K_{f} x_{i}$. Let $\mathscr{F}_{i}$ be a Borel measurable fundamental domain for the right action of $\Gamma_{i}$ on $G_{\infty}$. Here we mean a fundamental domain in the strict sense, that is, $\mathscr{F}_{i}$ is a set of representatives for $G_{\infty} / \Gamma_{i}$ (for the 
existence of measurable fundamental domains see [Bourbaki 1963, Chapter VII §2, Example 12]). The set $\mathscr{F}_{F}$ defined as the union $\bigsqcup_{i=1}^{m} \mathscr{F}_{i} K_{f} x_{i} \subseteq G(\mathbb{A})$ is a Borel measurable fundamental domain for the right action of $G(F)$ on $G(\mathbb{A})$. We write

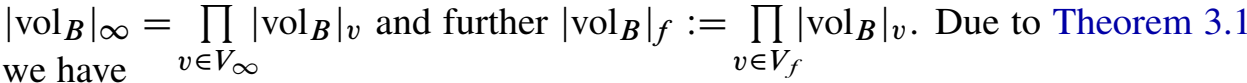

$$
\chi\left(K_{\infty} K_{f} \backslash G(\mathbb{A}) / G(F)\right)=\sum_{i=1}^{m} \chi\left(X / \Gamma_{i}\right)=\lambda \sum_{i=1}^{m} \int_{\mathscr{F}_{i}}\left|\operatorname{vol}_{B}\right|_{\infty},
$$

where

$$
\lambda=(-1)^{p} \frac{\left|W\left(\mathfrak{g}_{\infty, \mathbb{C}}\right)\right|}{\left|\pi_{0}\left(G_{\infty}\right)\right|\left|W\left(\mathfrak{k}_{\mathbb{C}}\right)\right|} \operatorname{vol}_{B}\left(G_{u}\right)^{-1}
$$

By multiplication with the volume of $K_{f}$, which is simply $\operatorname{vol}_{B}\left(K_{f}\right)=\int_{K_{f}}\left|\operatorname{vol}_{B}\right|{ }_{f}$, and by Lemma 3.2, we obtain

$$
\sum_{i=1}^{m} \int_{\mathscr{F}_{i}}\left|\operatorname{vol}_{B}\right|_{\infty} \operatorname{vol}_{B}\left(K_{f}\right)=\int_{\mathscr{F}} \prod_{v \in V}\left|\operatorname{vol}_{B}\right|_{v}=\left|d_{F}\right|^{d / 2} \int_{\mathscr{F}_{F}} \tau=\left|d_{F}\right|^{d / 2} \tau(G) .
$$

This means we have

$$
\chi\left(K_{\infty} K_{f} \backslash G(\mathbb{A}) / G(F)\right)=\lambda\left|d_{F}\right|^{d / 2} \tau(G) \operatorname{vol}_{B}\left(K_{f}\right)^{-1} .
$$

Finally we are left with the task of determining $\operatorname{vol}_{B}\left(K_{f}\right)$. We shall exploit that $K_{f}$ is given by the local datum $(U, \alpha)$. Since $\operatorname{vol}_{B}\left(K_{f}\right)=\prod_{v \in V_{f}} \operatorname{vol}_{B}\left(K_{v}(U)\right)$ and the scheme $G$ is smooth, we can apply a theorem of Weil (for a modern formulation see [Oesterlé 1984, Section I.2.5] or [Batyrev 1999, Theorem 2.5]) in every finite place to deduce

$$
\operatorname{vol}_{B}\left(K_{f}\right)=\prod_{v \in V_{f}} m(B)_{v} \frac{\left|U_{v}\right|}{\mathrm{N}\left(\mathfrak{p}_{v}\right)^{d \alpha_{v}}}
$$

Now the claim follows readily.

\section{Rohlfs' method}

In this section we give a short summary of Rohlfs' method for the computation of Lefschetz numbers.

Let $F$ be an algebraic number field and let $G$ be a linear algebraic group defined over $F$. We assume that $G$ has strong approximation. For example, unipotent groups and $F$-simple, simply connected groups with a noncompact associated Lie group have strong approximation [Platonov and Rapinchuk 1994, page 427]. Choose a maximal compact subgroup $K_{\infty} \subseteq G_{\infty}$ and set $X:=K_{\infty} \backslash G_{\infty}$. Furthermore, 
let $K_{f} \subseteq G\left(\mathbb{A}_{f}\right)$ be an open compact subgroup and let $\Gamma:=G(F) \cap K_{f}$ be the arithmetic group defined by this open compact subgroup. There is a homeomorphism

$$
X / \Gamma \stackrel{\simeq}{\longrightarrow} K_{\infty} K_{f} \backslash G(\mathbb{A}) / G(F) .
$$

To see this, consider the inclusion $G_{\infty} \rightarrow G(\mathbb{A})$ and use strong approximation to observe that it factors to such a homeomorphism. Recall that $\Gamma$ is torsion-free if and only if $G(F)$ acts freely on $K_{\infty} K_{f} \backslash G(\mathbb{A})$.

Let $\tau$ be an automorphism of finite order of $G$. We can choose $K_{\infty}$ such that it is $\tau$-stable. We further assume that $K_{f} \subset G\left(\mathbb{A}_{f}\right)$ is a $\tau$-stable open compact subgroup. We obtain an action of $\tau$ on the double coset space

$$
S\left(K_{f}\right):=K_{\infty} K_{f} \backslash G(\mathbb{A}) / G(F) .
$$

We describe the set $S\left(K_{f}\right)^{\tau}$ of $\tau$-fixed points following [Rohlfs 1990] under the assumption that $G(F)$ acts freely on $K_{\infty} K_{f} \backslash G(\mathbb{A})$.

Consider the finite set $\mathscr{H}^{1}(\tau)$ defined as the fibred product

$$
\mathscr{H}^{1}(\tau):=H^{1}\left(\tau, K_{\infty} K_{f}\right) \underset{H^{1}(\tau, G(\mathbb{A}))}{\times} H^{1}(\tau, G(F))
$$

of nonabelian cohomology sets. Here we usually write $\tau$ instead of the finite group $\langle\tau\rangle$ generated by $\tau$. We consider $\mathscr{H}^{1}(\tau)$ as a topological space with the discrete topology. Rohlfs [1990, Section 3.5] constructed a surjective and continuous map

$$
\vartheta: S\left(K_{f}\right)^{\tau} \rightarrow \mathscr{H}^{1}(\tau)
$$

In particular the fibres are open and closed in $S\left(K_{f}\right)^{\tau}$ and we get a decomposition

$$
S\left(K_{f}\right)^{\tau}=\bigsqcup_{\eta \in \mathscr{H}^{1}(\tau)} \vartheta^{-1}(\eta)
$$

Let $\gamma \in Z^{1}(\tau, G(F))$ be a cocycle. The $\gamma$-twisted $\tau$-action on $G$, defined by $\tau \mid \gamma(x)=\gamma_{\tau}^{\tau} x \gamma_{\tau}^{-1}$, is an automorphism defined over $F$ and the group of fixed points is a linear algebraic group which will be denoted $G(\gamma)$. Similarly, given a cocycle $k \in Z^{1}\left(\tau, K_{\infty} K_{f}\right)$ we define the $k$-twisted action of $\tau$ on $K_{\infty} K_{f}$ by $\tau \mid k_{g}:=k_{\tau} \tau_{g} k_{\tau}^{-1}$. The corresponding group of fixed points under this action will be written $\left(K_{\infty} K_{f}\right)^{\tau \mid k}$. Rohlfs obtained the following description of the fibres of $\vartheta$.

Lemma 4.1 [Rohlfs 1990, Section 3.5]. Let $K_{f} \subset G\left(\mathbb{A}_{f}\right)$ be a $\tau$-stable open compact subgroup such that $G(F)$ acts freely on $K_{\infty} K_{f} \backslash G(\mathbb{A})$.

Let $\eta \in \mathscr{H}^{1}(\tau)$ be a class represented by a pair of cocycles $(k, \gamma)$ with $\left(k_{s}\right)_{s}$ in $Z^{1}\left(\tau, K_{\infty} K_{f}\right)$ and $\left(\gamma_{s}\right)_{s} \in Z^{1}(\tau, G(F))$. Take $a \in G(\mathbb{A})$ such that ${ }^{s} a=k_{s}^{-1} a \gamma_{s}$ for all $s \in\langle\tau\rangle$. There is a homeomorphism

$$
a^{-1}\left(K_{\infty} K_{f}\right)^{\tau \mid k} a \backslash G(\gamma)(\mathbb{A}) / G(\gamma)(F) \stackrel{\simeq}{\longrightarrow} \vartheta^{-1}(\eta) .
$$


Combined with Theorem 4.2 below this yields a method for the computation of Lefschetz numbers which we simply call Rohlfs' method.

Definition. Let $\rho: G \rightarrow \operatorname{GL}(W)$ be a rational representation defined over the algebraic closure $\bar{F}$ of $F$. Here $W$ is a finite dimensional $\bar{F}$-vector space. Given an action of the finite group $\langle\tau\rangle$ on $W$, we say that this action is compatible with $\rho$ if

$$
{ }^{s}(\rho(g) v)=\rho\left({ }^{s} g\right){ }^{s} v
$$

for all $v \in V, s \in\langle\tau\rangle$ and $g \in G(\bar{F})$. In other words $W$ is a $(G(\bar{F}) \rtimes\langle\tau\rangle)$-module.

Let $\rho: G \rightarrow \mathrm{GL}(W)$ be a rational representation and let $\Gamma \subseteq G(F)$ be a torsionfree arithmetic subgroup. If $W$ is equipped with a compatible $\tau$-action then we define the Lefschetz number of $\tau$ with values in $W$ as

$$
\mathscr{L}(\tau, \Gamma, W):=\sum_{i=0}^{\infty}(-1)^{i} \operatorname{Tr}\left(\tau^{i}: H^{i}(\Gamma, W) \rightarrow H^{i}(\Gamma, W)\right) .
$$

Since torsion-free arithmetic groups are of type (FL), this is a finite sum.

Given a cocycle $b=\left(b_{s}\right)_{s} \in H^{1}(\tau, G(\bar{F}))$ one can define the $b$-twisted $\tau$-action on $W$ by

$$
\tau \mid b_{w}=b_{\tau} \tau_{w}
$$

for all $w \in W$. We write $W(b)$ to denote the space $W$ with the $b$-twisted $\tau$-action. We need the following slight paraphrase of a theorem of Rohlfs.

Theorem 4.2 [Rohlfs 1990]. Let $G$ be an algebraic $F$-group with strong approximation and let $\tau$ be an automorphism of finite order defined over $F$. Let $K_{f} \subset G\left(\mathbb{A}_{f}\right)$ be a $\tau$-stable open compact subgroup such that $\Gamma:=G(F) \cap K_{f}$ is torsion-free. Let $\rho: G \rightarrow \mathrm{GL}(W)$ be a rational representation defined over $\bar{F}$ with a compatible $\tau$-action. Then we have

$$
\mathscr{L}(\tau, \Gamma, W)=\sum_{\eta \in \mathscr{H}^{1}(\tau)} \chi\left(\vartheta^{-1}(\eta)\right) \operatorname{Tr}\left(\tau \mid W\left(b_{\eta}\right)\right),
$$

where $b_{\eta} \in G(F)$ is any representative of the $H^{1}(\tau, G(F))$ component of $\eta$.

Proof. This follows from Rohlfs' decomposition — see (2) — and a suitable Lefschetz fixed point principle — for instance [Rohlfs and Schwermer 1998, §2.3] or [Kionke 2012].

\section{Proof of the main theorem}

5A. Introduction. In this section we compute the Lefschetz number of an involution of symplectic type on principal congruence subgroups of inner forms of the special linear group. For this purpose we combine the tools developed in the previous sections. 
One should keep in mind that the central result is the adelic Lefschetz number formula in Theorem 4.2. Whenever we want to apply this theorem, there are two important steps to do. First step: understand the involved first nonabelian Galois cohomology sets. Second step: compute the Euler characteristics of the fixed point components. In the second step we use the adelic formula in Theorem 3.3 obtained from Harder's Gauss-Bonnet theorem.

First we introduce some notation, then we begin to determine various nonabelian cohomology sets. In the third subsection we describe the fixed point groups and we compute their Euler characteristics. Finally we prove the main theorem.

As before $F$ denotes an algebraic number field and $O$ denotes its ring of integers. Let $D$ be a quaternion algebra over $F$, that is, a central simple $F$-algebra of dimension four. Note that even though we use the symbol $D$, the quaternion algebra $D$ is in general not assumed to be a division algebra. Given a place $v$, we define $D_{v}:=D \otimes_{F} F_{v}$. If $D_{v}$ is isomorphic to $M_{2}\left(F_{v}\right)$, we say that $D$ splits at the place $v$. Otherwise $D_{v}$ is a division algebra and we say that $D$ is ramified at $v$. Let $\operatorname{Ram}(D) \subset V$ be the finite set of places where $D$ ramifies, and let $\operatorname{Ram}_{f}(D)$ (resp. $\left.\operatorname{Ram}_{\infty}(D)\right)$ denote the subset of finite (resp. Archimedean) places.

Definition. The signed reduced discriminant $\Delta_{\mathrm{rd}}(D)$ of $D$ is the integer

$$
\Delta_{\mathrm{rd}}(D):=(-1)^{r} \prod_{\mathfrak{p} \in \operatorname{Ram}_{f}(D)} \mathrm{N}(\mathfrak{p})
$$

where $r=\left|\operatorname{Ram}_{\infty}(D)\right|$.

5A1. The canonical involution. On the quaternion algebra $D$ we have the canonical involution

$$
\tau_{c}: D \rightarrow D, \quad \tau_{c}(x)=: \bar{x},
$$

sometimes called conjugation. Given a description $D=Q(a, b \mid F)$ of $D$ with $a, b \in F^{\times}$- meaning there is a basis $1, i, j, i j$ of $D$ with $i^{2}=a, j^{2}=b$ and $i j=-j i-$ conjugation is defined by

$$
\tau_{c}: x_{0}+x_{1} i+x_{2} j+x_{3} i j \mapsto x_{0}-x_{1} i-x_{2} j-x_{3} i j .
$$

Note that the conjugation is $F$-linear; that is, it is an involution of the first kind on $D$. Moreover, $\tau_{c}$ is an involution of symplectic type.

The elements fixed by conjugation are precisely the elements of $F$. The reduced norm and trace of $D$ are related to conjugation by

$$
\operatorname{trd}_{D}(x)=x+\bar{x}, \quad \operatorname{nrd}_{D}(x)=x \bar{x}=\bar{x} x
$$

for all $x \in D$. 
5A2. Orders. Let $\Lambda_{D}$ be an 0 -order in $D$. We show that $\Lambda_{D}$ is $\tau_{c}$-stable: let $x \in \Lambda_{D}$, then

$$
\bar{x}=x+\bar{x}-x=\operatorname{trd}_{D}(x)-x .
$$

Recall that $\operatorname{trd}_{D}(x) \in \mathbb{O}$ because $x$ is integral. Since $0 \subseteq \Lambda_{D}$, we obtain $\bar{x} \in \Lambda_{D}$. Moreover, it follows directly from the definitions that $\Lambda_{D}$ is smooth if and only if $\Lambda_{D}$ is $\tau_{c}$-smooth (see the second definition on page 376, and the one on page 378).

We will assume from now on that $\Lambda_{D}$ is a maximal 0 -order in $D$. In particular, it is a smooth and $\tau_{c}$-smooth order (see Proposition 2.4).

Let $n$ be a positive integer. Consider the central simple $F$-algebra

$$
A:=M_{n}(D)
$$

of $n \times n$-matrices with entries in the quaternion algebra $D$. The canonical involution on $D$ induces an involution $\tau$ on $A$ defined by

$$
\tau(x):={ }^{\tau} x:=\bar{x}^{T}
$$

that is, conjugate every entry in the matrix $x$ and then transpose the matrix. It is easily checked that this defines an involution of symplectic type on $A$ [Knus et al. 1998, Proposition 2.23].

Lemma 5.1. Let $\Lambda_{D} \subseteq D$ be a maximal O-order. The O-order $\Lambda=M_{n}\left(\Lambda_{D}\right)$ in $A$ is maximal, $\tau$-stable, smooth and $\tau$-smooth.

Proof. Since $\Lambda_{D}$ is stable under conjugation, it is obvious that $\Lambda$ is $\tau$-stable. Moreover, it follows from [Reiner 2003, Theorem (21.6)] that $\Lambda$ is a maximal O-order. In turn Proposition 2.4 shows that $\Lambda$ is also a smooth order.

Finally we need to check that $\Lambda$ is $\tau$-smooth. Let $x \in \operatorname{Sym}(\Lambda, \tau)$ be an element which is fixed by $\tau$. This means that $x=\left(x_{i j}\right)$ satisfies

$$
x_{i j}=\bar{x}_{j i} \quad \text { for all } i \neq j
$$

and

$$
x_{i i} \in \mathcal{O} \text {. }
$$

The order $\Lambda_{D}$ is smooth, therefore there is, for every $i=1, \ldots, n$, an element $z_{i} \in \Lambda_{D}$ with $\operatorname{trd}_{D}\left(z_{i}\right)=z_{i}+\bar{z}_{i}=x_{i i}$. Now we define the upper triangular element $y \in \Lambda$ by

$$
y_{i j}:= \begin{cases}0 & \text { if } i>j, \\ x_{i j} & \text { if } i<j, \\ z_{i} & \text { if } i=j,\end{cases}
$$

and it is easy to see that $y+{ }^{\tau} y=x$. We deduce that $\Lambda$ is $\tau$-smooth. 
5A3. Setting and assumptions. We define $G:=\mathrm{SL}_{\Lambda}$ to be the special linear group over the order $\Lambda$ (see the first definition on page 376). From the previous lemma and Corollary 2.3 we deduce that $G$ is a smooth group scheme over $\mathbf{O}$. Moreover, the involution $\tau$ induces an automorphism $\tau^{*}$ of GL $\Lambda$ where $\tau^{*}=$ inv $\circ \tau$ (see Section 2C). Clearly $\tau^{*}$ has order (at most) two and restricts to an automorphism of $G=\mathrm{SL}_{\Lambda}$.

The real Lie group $G_{\infty}$ associated with $G$ is

$$
G_{\infty}:=\prod_{v \in V_{\infty}} G\left(F_{v}\right) \cong \mathrm{SL}_{2 n}(\mathbb{R})^{s} \times \mathrm{SL}_{n}(\mathbb{M})^{r} \times \mathrm{SL}_{2 n}(\mathbb{C})^{t}
$$

Here $s$ denotes the number real places of $F$ where $D$ splits, $r$ is the number of real places where $D$ ramifies, and $t$ is the number of complex places of $F$. The

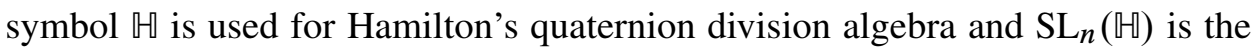
group of elements with reduced norm one in the central simple $\mathbb{R}$-algebra $M_{n}(\mathbb{W})$. Note that $[F: \mathbb{Q}]=r+s+2 t$. For every Archimedean place $v$ we fix a $\tau^{*}$ stable maximal compact subgroup $K_{v} \subseteq G\left(F_{v}\right)$; then the group $K_{\infty}:=\prod_{v \in V_{\infty}} K_{v}$ is
a $\tau^{*}$-stable maximal compact subgroup of $G_{\infty}$.

We study the cohomology of congruence subgroups arising from the group $\mathrm{SL}_{\Lambda}$. Let $\mathfrak{a} \subseteq \mathcal{O}$ be a proper ideal; we define the principal congruence subgroup

$$
\Gamma(\mathfrak{a}):=\operatorname{ker}(G(\mathcal{O}) \rightarrow G(\mathcal{O} / \mathfrak{a}))
$$

of level $\mathfrak{a}$. We shall always assume that $\Gamma(\mathfrak{a})$ is torsion-free (which holds for almost all ideals). Note that the groups $\Gamma(\mathfrak{a})$ are always $\tau^{*}$-stable.

These groups can be described by local data. Let $\mathfrak{p} \subseteq \mathcal{O}$ be a prime ideal of 0 and let $v$ be the associated finite place. Let $v_{\mathfrak{p}}(\mathfrak{a})$ be the maximal exponent $e$ such that $\mathfrak{p}^{e}$ divides $\mathfrak{a}$; then $\mathfrak{a} O_{v}=\mathfrak{p}^{v_{\mathfrak{p}}(\mathfrak{a})} \mathscr{O}_{v}$. We obtain an open and compact subgroup $K_{v} \subseteq G\left(O_{v}\right)$ defined as

$$
K_{v}:=\operatorname{ker}\left(G\left(\mathrm{O}_{v}\right) \longrightarrow G\left(\mathbb{O}_{v} / \mathfrak{a} \mathfrak{O}_{v}\right)\right) .
$$

We form the direct product $K_{f}:=\prod_{v \in V_{f}} K_{v}$, which is an open and compact subgroup of the locally compact group $G\left(\mathbb{A}_{f}\right)$. Clearly, $\Gamma(\mathfrak{a})=G(F) \cap K_{f}$.

We keep the notation introduced in this section. We always assume that

(i) the order $\Lambda_{D}$ is a maximal order in $D$, and

(ii) the ideal $\mathfrak{a} \subseteq 0$ is nontrivial and chosen such that $\Gamma(\mathfrak{a})$ is torsion-free.

5B. Hermitian forms and Galois cohomology. In this section we determine the nonabelian Galois cohomology set $\mathscr{H}^{1}\left(\tau^{*}\right)$. Recall that $\mathscr{H}^{1}\left(\tau^{*}\right)$ is the fibred product

$$
\mathscr{H}^{1}\left(\tau^{*}\right):=H^{1}\left(\tau^{*}, K_{\infty} K_{f}\right) \underset{H^{1}\left(\tau^{*}, G(\mathrm{~A})\right)}{\times} H^{1}\left(\tau^{*}, G(F)\right) .
$$


In order to determine this set we need to calculate local and global cohomology sets. The global problem is to determine $H^{1}\left(\tau^{*}, G(F)\right)$, whereas locally we have to calculate $H^{1}\left(\tau^{*}, G\left(F_{v}\right)\right)$ and $H^{1}\left(\tau^{*}, K_{v}\right)$ for every place $v$. We start by determining the corresponding cohomology sets for $\mathrm{GL}_{\Lambda}$. This task amounts to the classification of certain hermitian forms over quaternion algebras, which is well known (see for instance [Shimura 1963, §2] or [Scharlau 1985, Chapter 10]). Afterwards we use the pfaffian to obtain results for the special linear group.

5B1. Local results for $\mathrm{GL}_{\Lambda}$. We introduce the following notation: given two integers $p, q \geq 0$, we define the diagonal matrix

$$
I_{p, q}=\operatorname{diag}(\underbrace{1, \ldots, 1}_{p}, \underbrace{-1, \ldots,-1}_{q}) .
$$

Proposition 5.2. Let $v \in V$ be a place of $F$. If $v$ is a real place where $D$ is ramified, then

$$
H^{1}\left(\tau^{*}, \mathrm{GL}_{\Lambda}\left(F_{v}\right)\right) \cong\left\{I_{p, q} \mid p, q \geq 0 \text { with } p+q=n\right\} .
$$

This means that the matrices $I_{p, q}$ are a system of representatives for the cohomology classes. The cohomology is trivial for all places $v \in V \backslash \operatorname{Ram}_{\infty}(D)$, that is,

$$
H^{1}\left(\tau^{*}, \operatorname{GL}_{\Lambda}\left(F_{v}\right)\right)=\{1\} .
$$

Proof. Let $b \in Z^{1}\left(\tau^{*}, \mathrm{GL}_{\Lambda}\left(F_{v}\right)\right)$ be a cocycle; $b$ is an element of $\operatorname{GL}_{n}\left(D_{v}\right)$ satisfying $b={ }^{\tau} b$. Such a matrix $b$ defines a regular hermitian form on the free right $D_{v}$-module $D_{v}^{n}$.

If $v \in V \backslash \operatorname{Ram}_{\infty}(D)$ (i.e., $v$ is not a real ramified place), regular hermitian forms over $D_{v}$ are classified by their dimension over $F_{v}$; this follows from [Scharlau 1985, Chapter 10, Theorem 1.7 and Example 1.8]. Note that these results cover the case where $D_{v}$ is a division algebra. However it is easy to obtain an analogous result if $D_{v} \cong M_{2}\left(F_{v}\right)$ (at least for free regular hermitian spaces). Thus we find $g \in \mathrm{GL}_{n}\left(D_{v}\right)$ with $g b^{\tau} g=1$, and so the second assertion follows immediately.

Let $v \in \operatorname{Ram}_{\infty}(D)$; then $D_{v} \cong \mathbb{H}$. In this case $\tau_{c}$-hermitian forms are classified by dimension and signature. Translated to the setting of nonabelian Galois cohomology, this means that the set $\left\{I_{p, q} \mid p, q \geq 0\right.$ with $\left.p+q=n\right\}$ is a system of representatives for $H^{1}\left(\tau^{*}, \mathrm{GL}_{\Lambda}\left(F_{v}\right)\right)$.

Definition. Let $v \in \operatorname{Ram}_{\infty}(D)$. For a cocycle $b \in Z^{1}\left(\tau^{*}, \mathrm{GL}_{\Lambda}\left(F_{v}\right)\right)$ which is cohomologous to $I_{p, q}$ we say that the signature of $b$ is the pair $(p, q)$.

Corollary 5.3. Let $v \in V_{f}$ be a finite place; then $H^{1}\left(\tau^{*}, \mathrm{GL}_{\Lambda}\left(\mathrm{O}_{v}\right)\right)=\{1\}$.

Proof. The 0 -order $\Lambda$ is maximal and $\tau$-smooth (see Lemma 5.1) and the same holds for the $\mathscr{O}_{v}$-order $\Lambda \otimes \mathcal{O}_{v}$ (see [Reiner 2003, Corollary (11.6)] and note that 
$\tau$-smoothness is a local property). By Theorem 2.10 (Fainsilber and Morales), the canonical map

$$
H^{1}\left(\tau^{*}, \mathrm{GL}_{\Lambda}\left(\mathrm{O}_{v}\right)\right) \rightarrow H^{1}\left(\tau^{*}, \mathrm{GL}_{\Lambda}\left(F_{v}\right)\right)
$$

is injective, and hence the assertion follows immediately from Proposition 5.2.

5B2. Global results for $\mathrm{GL}_{\Lambda}$. We analyse $H^{1}\left(\tau^{*}, \mathrm{GL}_{\Lambda}(F)\right)$, again using the classification of $\tau_{c}$-hermitian forms.

Proposition 5.4 (Hasse principle). The canonical map

$$
H^{1}\left(\tau^{*}, \mathrm{GL}_{\Lambda}(F)\right) \longrightarrow \prod_{v \in \operatorname{Ram}_{\infty}(D)} H^{1}\left(\tau^{*}, \mathrm{GL}_{\Lambda}\left(F_{v}\right)\right)
$$

induced by the inclusions is bijective. This means a class in $H^{1}\left(\tau^{*}, \mathrm{GL}_{\Lambda}(F)\right)$ is uniquely determined by its signatures at the real ramified places.

Proof. If $D$ is not a division algebra it is easily checked that $H^{1}\left(\tau^{*}, \operatorname{GL}_{\Lambda}(F)\right)=\{1\}$. Thus there is nothing to show.

Assume that $D$ is a division algebra. The regular hermitian forms over $D$ (with respect to $\tau_{c}$ ) are classified by dimension and their signatures at the real places of $F$ where $D$ ramifies [Scharlau 1985, Chapter 10, Example 1.8]. The claim follows as in the local case.

5B3. The pfaffian associated with $\tau$. We explain how to compute the pfaffian associated with $\tau$ (see Section 2D1) for diagonal matrices. Let $k$ be any extension field of $F$, for example a local completion. Given a diagonal matrix $x=\operatorname{diag}\left(x_{1}, \ldots, x_{n}\right)$ with entries in $k$, we can consider $x$ as a $\tau$-fixed matrix in $A \otimes_{F} k=M_{n}\left(D \otimes_{F} k\right)$. Lemma 5.5. For $x=\operatorname{diag}\left(x_{1}, \ldots, x_{n}\right)$ with entries in some extension field $k$ of $F$, the pfaffian of $x$ is the product of all entries:

$$
\operatorname{pf}_{\tau}(x)=x_{1} x_{2} \cdots x_{n} .
$$

Proof. We can assume without loss of generality that $k$ is algebraically closed. In this case $D \otimes_{F} k \cong M_{2}(k)$ and the reduced norm $\operatorname{nrd}_{D}: D \otimes_{F} k \rightarrow k$ agrees with the determinant, in particular it is surjective. This means, for given $i \in\{1, \ldots, n\}$, we can write $x_{i}=\operatorname{nrd}_{D}\left(y_{i}\right)=\bar{y}_{i} y_{i}$ for some $y_{i} \in D \otimes_{F} k$. Consider the matrix $y=\operatorname{diag}\left(y_{1}, \ldots, y_{n}\right) \in M_{n}\left(D \otimes_{F} k\right)$ : it satisfies $\tau(y) y=x$. By Lemma 2.7 we obtain

$$
\operatorname{pf}_{\tau}(x)=\operatorname{nrd}_{A}(y)=\prod_{i=1}^{n} \operatorname{nrd}_{D}\left(y_{i}\right)=\prod_{i=1}^{n} x_{i} .
$$

Here we used that the reduced norm of a diagonal matrix in $M_{n}\left(D \otimes_{F} k\right)$ is the product of the reduced norms of the entries; see [Weil 1995, Chapter IX, §2, Corollary 2].

Note in particular that the pfaffian $\operatorname{pf}_{\tau}: \operatorname{Sym}(\Lambda, \tau) \rightarrow 0$ is surjective. 
5B4. Transfer of results to $\mathrm{SL}_{\Lambda}$. The final step in this section is to transfer the results on nonabelian Galois cohomology with values in $\mathrm{GL}_{\Lambda}$ to the group $G=\mathrm{SL}_{\Lambda}$. Our main tool is the cohomological diagram for symplectic involutions.

Lemma 5.6. Let $v \in V \backslash \operatorname{Ram}_{\infty}(D)$ be a place of $F$. Then the pfaffian induces a bijection

$$
\operatorname{pf}_{\tau}: H^{1}\left(\tau^{*}, \operatorname{SL}_{\Lambda}\left(F_{v}\right)\right) \stackrel{\simeq}{\longrightarrow}\{ \pm 1\} .
$$

Proof. If follows from Proposition 5.2 that $H^{1}\left(\tau^{*}, \mathrm{GL}_{\Lambda}\left(F_{v}\right)\right)$ is trivial. The cohomological diagram for symplectic involutions (see Proposition 2.8) collapses to

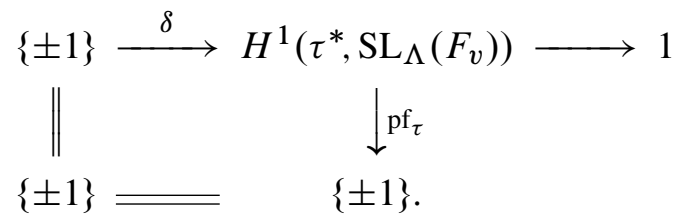

Here we used that $\operatorname{nrd}_{\Lambda}: \mathrm{GL}_{\Lambda}\left(F_{v}\right) \rightarrow F_{v}^{\times}$is surjective, see [Reiner 2003, Theorem (33.4)]. By Proposition 2.8 the morphism $\delta$ is injective, and thus bijective.

Lemma 5.7. Let $v \in \operatorname{Ram}_{\infty}(D)$. The canonical map

$$
j_{*}: H^{1}\left(\tau^{*}, \mathrm{SL}_{\Lambda}\left(F_{v}\right)\right) \rightarrow H^{1}\left(\tau^{*}, \mathrm{GL}_{\Lambda}\left(F_{v}\right)\right)
$$

is bijective.

Proof. In this case the reduced norm takes only positive values in $F_{v} \cong \mathbb{R}$. Therefore the cohomological diagram for symplectic involutions (Proposition 2.8) yields

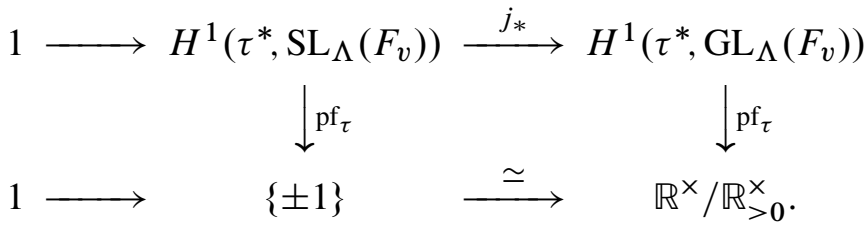

It follows directly from Corollary 2.9 that $j_{*}$ is surjective. Moreover, twisting the upper row with cocycles for $H^{1}\left(\tau^{*}, \mathrm{SL}_{\Lambda}\left(F_{v}\right)\right)$ shows that $j_{*}$ is indeed injective. For more details on twisting in nonabelian cohomology the reader may consult [Serre 1994, Chapter I, §5.4]. Note that twisting an involution of symplectic type gives an involution of symplectic type (see the remark on page 381 ).

Lemma 5.8. Let $v$ be a finite place and let $\mathfrak{p}_{v} \subseteq \mathrm{O}_{v}$ be the prime ideal. For an integer $m \geq 0$ we define $K_{v}(m):=\operatorname{ker}\left(G\left(\mathcal{O}_{v}\right) \rightarrow G\left(\mathcal{O}_{v} / \mathfrak{p}_{v}^{m}\right)\right)$. Then the pfaffian induces a bijection

$$
\operatorname{pf}_{\tau}: H^{1}\left(\tau^{*}, K_{v}(m)\right) \stackrel{\simeq}{\longrightarrow} \begin{cases}\{ \pm 1\} & \text { if }-1 \equiv 1 \quad \bmod \mathfrak{p}_{v}^{m}, \\ \{1\} & \text { otherwise. }\end{cases}
$$


Proof. We start with the special case $m=0$; here $K_{v}(m)=\mathrm{SL}_{\Lambda}\left(\mathrm{O}_{v}\right)$. Here the claim follows just as in the proof of Lemma 5.6 from Proposition 2.8, Corollary 5.3, and the fact that the reduced norm $\operatorname{nrd}_{\Lambda}: \mathrm{GL}_{\Lambda}\left(\mathrm{O}_{v}\right) \rightarrow \mathrm{O}_{v}^{\times}$is onto [Reiner 2003, Theorem (14.1) and Exercise 5 on page 152].

For $m \geq 1$ consider the short exact sequence of groups

$$
1 \longrightarrow K_{v}(m) \longrightarrow \mathrm{SL}_{\Lambda}\left(\mathrm{O}_{v}\right) \longrightarrow \mathrm{SL}_{\Lambda}\left(\mathrm{O}_{v} / \mathfrak{p}_{v}^{m}\right) \longrightarrow 1
$$

Note that this sequence uses that the order $\Lambda$, and hence the group scheme $\mathrm{SL}_{\Lambda}$, is smooth by Lemma 5.1. We obtain a long exact sequence of pointed sets

$$
G^{\tau^{*}}\left(\mathscr{O}_{v}\right) \stackrel{\pi}{\longrightarrow} G^{\tau^{*}}\left(\mathcal{O}_{v} / \mathfrak{p}_{v}^{m}\right) \stackrel{\delta}{\longrightarrow} H^{1}\left(\tau^{*}, K_{v}(m)\right) \stackrel{j_{m}}{\longrightarrow} H^{1}\left(\tau^{*}, G\left(\mathscr{O}_{v}\right)\right) .
$$

It follows from the remark on page 379 that the fixed point group $G^{\tau^{*}}$ is just the group scheme $G(\Lambda, \tau)$ defined in Section $2 C$. Since the group scheme $G(\Lambda, \tau)$ is smooth (Proposition 2.6), the canonical map $\pi$ is surjective, and so $\delta$ is trivial. Via twisting (see the remark on page 381) we obtain that $j_{m}$ is injective.

We use that the pfaffian is a morphism of schemes defined over 0 (as explained in Section 2D1). Given a cocycle $b \in Z^{1}\left(\tau^{*}, K_{v}(m)\right)$, we have $\operatorname{pf}_{\tau}(b) \equiv 1 \bmod \mathfrak{p}_{v}^{m}$. Consequently, if 1 and -1 are not congruent modulo $\mathfrak{p}_{v}^{m}$, then $H^{1}\left(\tau^{*}, K_{v}(m)\right)=\{1\}$ and the claim follows.

Assume now that $-1 \equiv 1 \bmod \mathfrak{p}_{v}^{m}$. Then the matrix $\operatorname{diag}(-1,1, \ldots, 1)$ lies in $K_{v}(m)$ and has pfaffian -1 (see Section 5B3).

For a real place $v \in V_{\infty}$ we denote the associated embedding $F \rightarrow \mathbb{R}$ by $\iota_{v}$. Define

$$
F_{D}^{\times}=\left\{x \in F^{\times} \mid \iota_{v}(x)>0 \text { for all } v \in \operatorname{Ram}_{\infty}(D)\right\} .
$$

By the Hasse-Schilling-Maass theorem [Reiner 2003, Theorem (33.15)] the image of the reduced norm $\operatorname{nrd}_{A}: A^{\times} \rightarrow F^{\times}$is $F_{D}^{\times}$.

Lemma 5.9. Assume that $\operatorname{Ram}_{\infty}(D)$ is not empty. Then the canonical morphism of pointed sets

$$
j_{*}: H^{1}\left(\tau^{*}, \mathrm{SL}_{\Lambda}(F)\right) \longrightarrow H^{1}\left(\tau^{*}, \mathrm{GL}_{\Lambda}(F)\right)
$$

is injective. The image consists of precisely those classes $x \in H^{1}\left(\tau^{*}, \mathrm{GL}_{\Lambda}(F)\right)$ which satisfy $\mathrm{pf}_{\tau}(x)= \pm 1 \cdot F_{D}^{\times}$.

If otherwise $D$ splits at every real place, then the pfaffian induces a bijection

$$
\operatorname{pf}_{\tau}: H^{1}\left(\tau^{*}, \mathrm{SL}_{\Lambda}(F)\right) \stackrel{\simeq}{\longrightarrow}\{ \pm 1\} .
$$

Proof. Assume that $\operatorname{Ram}_{\infty}(D)$ is empty. By the Hasse-Schilling-Maass theorem the reduced norm $\mathrm{GL}_{\Lambda}(F) \rightarrow F^{\times}$is surjective and the second assertion follows as in Lemma 5.6. 
Now we assume that $\operatorname{Ram}_{\infty}(D)$ is not empty. The image of the reduced norm $\operatorname{nrd}_{A}: A^{\times} \rightarrow F^{\times}$is $F_{D}^{\times}$. Note that $F_{D}^{\times}$cannot contain the element -1 since $\operatorname{Ram}_{\infty}(D)$ is not empty. Consider the cohomological diagram for symplectic involutions (Proposition 2.8)

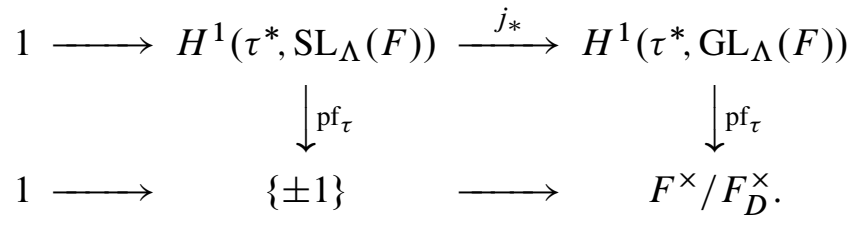

Twisting shows that the map $j_{*}$ is injective. The assertion about the image of $j_{*}$ follows immediately from Corollary 2.9 .

Remark. Assume that $\operatorname{Ram}_{\infty}(D)$ is not empty. Let $x \in H^{1}\left(\tau^{*}, \mathrm{GL}_{\Lambda}(F)\right)$ be a cohomology class. For every place $v \in \operatorname{Ram}_{\infty}(D)$ the class $x$ considered as a class in $H^{1}\left(\tau^{*}, \mathrm{GL}_{\Lambda}\left(F_{v}\right)\right)$ has a local signature $\left(p_{v}, q_{v}\right)$. Then according to Lemma 5.9 the class $x$ lies in the image of $j_{*}$ if and only if

$$
q_{v} \equiv q_{w} \quad \bmod 2
$$

for every pair of places $v, w \in \operatorname{Ram}_{\infty}(D)$. This means that either all $q_{v}$ are even or all $q_{v}$ are odd.

Theorem 5.10. Let $K_{f}=\prod_{v \in V_{f}} K_{v} \subseteq G\left(\mathbb{A}_{f}\right)$ be the open compact subgroup associated with the congruence subgroup $\Gamma(\mathfrak{a})$ (see Section 5A3). Consider the set $\mathscr{H}^{1}\left(\tau^{*}\right)$ (see the beginning of Section 5B). The projection $\pi: \mathscr{H}^{1}\left(\tau^{*}\right) \rightarrow$ $H^{1}\left(\tau^{*}, G(F)\right)$ is injective and there is a short exact sequence of pointed sets

$$
1 \longrightarrow \mathscr{H}^{1}\left(\tau^{*}\right) \stackrel{\pi}{\longrightarrow} H^{1}\left(\tau^{*}, G(F)\right) \stackrel{\mathrm{pf}_{\tau}}{\longrightarrow}\{ \pm 1\} \longrightarrow 1 .
$$

Proof. Consider the nonabelian cohomology set $H^{1}\left(\tau^{*}, K_{\infty} K_{f}\right)$, which agrees with the direct product $H^{1}\left(\tau^{*}, K_{\infty}\right) \times H^{1}\left(\tau^{*}, K_{f}\right)$. The canonical map

$$
H^{1}\left(\tau^{*}, K_{\infty}\right) \rightarrow H^{1}\left(\tau^{*}, G_{\infty}\right)
$$

is bijective (see [An and Wang 2008] or [Rohlfs 1981, Lemma 1.4]). Moreover, for every finite place $v \in V_{f}$ the group $K_{v}$ is of the form

$$
K_{v}(m)=\operatorname{ker}\left(G\left(\mathcal{O}_{v}\right) \rightarrow G\left(\mathcal{O}_{v} / \mathfrak{p}_{v}^{m}\right)\right)
$$

for some integer $m$. It follows from Lemma 5.8 that the inclusion $K_{v} \rightarrow G\left(F_{v}\right)$ induces an injection $H^{1}\left(\tau^{*}, K_{v}\right) \rightarrow H^{1}\left(\tau^{*}, G\left(F_{v}\right)\right)$. Therefore the canonical map $H^{1}\left(\tau^{*}, K_{\infty} K_{f}\right) \rightarrow H^{1}\left(\tau^{*}, G(\mathbb{A})\right)$ is injective and we conclude that the projection $\pi: \mathscr{H}^{1}\left(\tau^{*}\right) \rightarrow H^{1}\left(\tau^{*}, G(F)\right)$ is injective.

Moreover, it follows from the considerations on diagonal matrices in Section 5B3 that the pfaffian $\mathrm{pf}_{\tau}: H^{1}\left(\tau^{*}, G(F)\right) \rightarrow\{ \pm 1\}$ is surjective. 
It remains to understand the image of $\pi$. Since $\Gamma(\mathfrak{a})$ is (by assumption) torsionfree, we know that -1 is not congruent to 1 modulo $\mathfrak{a}$. In particular there is a prime ideal $\mathfrak{p}$ which divides $\mathfrak{a}$, say $e=v_{\mathfrak{p}}(\mathfrak{a})$, such that 1 and -1 are not congruent modulo $\mathfrak{p}^{e}$. Let $v \in V_{f}$ be the finite place associated with $\mathfrak{p}$, then $K_{v}=K_{v}(e)$ and $H^{1}\left(\tau^{*}, K_{v}\right)=\{1\}$ by Lemma 5.8. Let $\gamma \in H^{1}\left(\tau^{*}, G(F)\right)$ be in the image of $\pi$, say $(x, \gamma)$ is the inverse image in $\mathscr{H}^{1}\left(\tau^{*}\right)$. Let $x_{v}$ be the projection of the class $x$ to $H^{1}\left(\tau^{*}, K_{v}\right)$. Since $x$ and $\gamma$ have the same image in $H^{1}\left(\tau^{*}, G(\mathbb{A})\right)$, we can deduce that $\operatorname{pf}_{\tau}(\gamma)=\mathrm{pf}_{\tau}\left(x_{v}\right)=1$.

Conversely, given $\gamma \in H^{1}\left(\tau^{*}, G(F)\right)$ in the kernel of the pfaffian, then $\gamma$ lies in the image of $\pi$. Let $c_{\infty} \in H^{1}\left(\tau^{*}, K_{\infty}\right)$ be a cohomology class such that $c_{\infty}$ and $\gamma$ define the same class in $H^{1}\left(\tau^{*}, G_{\infty}\right)$. Let $1_{f}$ denote the trivial class in $H^{1}\left(\tau^{*}, K_{f}\right)$, then the triple $\left(c_{\infty}, 1_{f}, \gamma\right)$ is a class in $\mathscr{H}^{1}\left(\tau^{*}\right)$ which is mapped to $\gamma$ by $\pi$.

5C. The fixed point groups. Up to Section 5C6 the number field $F$ is assumed to be totally real.

Definition. Let $R$ be a commutative 0 -algebra (for example $\mathcal{O}_{v}$ or $F_{v}$ ). For every cocycle $\gamma$ in $Z^{1}\left(\tau^{*}, G(R)\right)$ the $R$-group scheme $G(\gamma)$ of $\tau^{*} \mid \gamma$-fixed points is defined by

$$
G(\gamma)(C):=\left\{g \in G(C)\left|g=\tau^{*}\right| \gamma g\right\}
$$

for any commutative $R$-algebra $C$. Recall that the $\gamma$-twisted $\tau^{*}$-action is given by $\tau^{*} \mid \gamma_{g}=\gamma_{g}^{*} \gamma^{-1}$.

We define the symplectic group $\mathrm{Sp}_{n}$ over $\mathbb{Z}$ by

$$
\mathrm{Sp}_{n}(R):=\left\{g \in \mathrm{GL}_{2 n}(R) \mid g^{T} J g=J\right\},
$$

for every commutative ring $R$, where $J$ is the standard symplectic matrix

$$
J=\left(\begin{array}{rr}
0_{n} & 1_{n} \\
-1_{n} & 0_{n}
\end{array}\right) .
$$

Note that in this notation $\mathrm{Sp}_{n}$ is of rank $n$, but consists of matrices of size $2 n \times 2 n$.

Given a cocycle $\gamma \in Z^{1}\left(\tau^{*}, G(0)\right)$, we want to understand the associated group scheme $G(\gamma)$. In particular we want to calculate the Euler characteristic of congruence subgroups of this group. We start with some basic observations and afterwards we collect all the ingredients necessary for an application of the adelic Euler characteristic formula (Theorem 3.3).

Remark. If $\gamma \in Z^{1}\left(\tau^{*}, G(\mathcal{O})\right)$ then $G(\gamma)=G(\Lambda, \tau \mid \gamma)$ in the notation of Section $2 \mathrm{C}$. The reason for this identity is that $G(\Lambda, \tau \mid \gamma)$ is always a closed subscheme of $\mathrm{SL}_{\Lambda}$, that is, all elements have reduced norm one (see the remark on page 379 ). Here $\tau \mid \gamma$ is the $\gamma$-twisted involution on $A$ (see the remark on page 381). Recall that 
$\tau \mid \gamma$ is of symplectic type, and that twisting and the operation $*$ commute, that is $(\tau \mid \gamma)^{*}=\tau^{*} \mid \gamma$.

Lemma 5.11. For every $\gamma \in Z^{1}\left(\tau^{*}, G(0)\right)$ the group scheme $G(\gamma)$ is smooth.

Proof. By Lemma 5.1 the order $\Lambda$ is $\tau$-smooth. The remark on page 381 implies further that $\Lambda$ is $(\tau \mid \gamma)$-smooth as well, and thus Proposition 2.6 yields that $G(\gamma)=G(\Lambda, \tau \mid \gamma)$ is smooth.

Lemma 5.12. Let $R$ be a commutative $\mathcal{O}$-algebra. Suppose the two cocycles $\gamma, \gamma^{\prime} \in$ $Z^{1}\left(\tau^{*}, G(R)\right)$ define the same class in $H^{1}\left(\tau^{*}, \mathrm{GL}_{\Lambda}(R)\right)$. Then $G(\gamma)$ and $G\left(\gamma^{\prime}\right)$ are isomorphic as group schemes over $R$.

Proof. There is $c \in \mathrm{GL}_{\Lambda}(R)$ satisfying $\gamma^{\prime}=c \gamma^{\tau} c$. We define a morphism of group schemes $f: G(\gamma) \rightarrow G\left(\gamma^{\prime}\right)$ by

$$
f_{C}: g \mapsto c g c^{-1}
$$

for every commutative $R$-algebra $C$ and all $g \in G(\gamma)(C)$. This map is well-defined:

$$
\tau^{*} \mid \gamma^{\prime}\left(c g c^{-1}\right)=\gamma^{\prime} \tau_{c}^{*} \tau_{g}^{*} \tau_{c}^{*-1} \gamma^{\prime-1}=c \gamma^{\tau_{g}^{*} \gamma^{-1} c^{-1}=c g c^{-1} .}
$$

Obviously the inverse map of $f$ is given by $g \mapsto c^{-1} g c$, so $f$ is an isomorphism.

Corollary 5.13. Let $\gamma \in Z^{1}\left(\tau^{*}, G(0)\right)$ be a cocycle, and let $R$ be a commutative O-algebra with $H^{1}\left(\tau^{*}, \mathrm{GL}_{\Lambda}(R)\right)=\{1\}$. There is an isomorphism of $R$-group schemes

$$
G(\gamma) \times_{\mathbb{O}} R \stackrel{\simeq}{\longrightarrow} G(1) \times_{\mathbb{O}} R .
$$

In particular, this holds if $R=0_{v}$ for $v \in V_{f}$ (see Corollary 5.3).

Moreover, if $k$ is a splitting field of $D$, then $G(\gamma) \times_{\mathbb{O}} k$ is isomorphic to the symplectic group $\mathrm{Sp}_{n} \times_{\mathbb{Z}} k$ defined over $k$.

Proof. The first part follows immediately from Lemma 5.12. For the second assertion note that we can choose a splitting $\varphi: A \otimes k \rightarrow M_{2 n}(k)$ such that $\varphi(\tau(x))$ equals $J \varphi(x)^{T} J^{-1}$, where $J$ denotes the standard symplectic matrix.

5C1. The associated real Lie groups. Let $\gamma \in Z^{1}\left(\tau^{*}, G(\mathcal{O})\right)$ be a cocycle. Consider the real Lie group

$$
G(\gamma)_{\infty}=\prod_{v \in V_{\infty}} G(\gamma)\left(F_{v}\right)
$$

associated with the group $G(\gamma)$.

Lemma 5.14. Let $\gamma \in Z^{1}\left(\tau^{*}, G(O)\right)$ be a cocycle and let $v \in \operatorname{Ram}_{\infty}(D)$ be a real ramified place. If the class of $\gamma$ in $H^{1}\left(\tau^{*}, G\left(F_{v}\right)\right)$ has signature $(p, q)$, then there is an isomorphism of real Lie groups

$$
G(\gamma)\left(F_{v}\right) \stackrel{\simeq}{\longrightarrow} \operatorname{Sp}(p, q) .
$$


Here $\operatorname{Sp}(p, q)$ is the real Lie group defined by

$$
\operatorname{Sp}(p, q):=\left\{g \in \mathrm{GL}_{n}(\mathbb{H}) \mid \bar{g}^{T} I_{p, q} g=I_{p, q}\right\} .
$$

Proof. This follows from Lemma 5.12 and the description of the cohomology set $H^{1}\left(\tau^{*}, \mathrm{GL}_{\Lambda}\left(F_{v}\right)\right)$ in Proposition 5.2.

For a real ramified place $v \in \operatorname{Ram}_{\infty}(D)$, let $\left(p_{v}, q_{v}\right)$ denote the local signature of the cohomology class of $\gamma$ in $H^{1}\left(\tau^{*}, G\left(F_{v}\right)\right)$. It follows from Corollary 5.13 and Lemma 5.14 that there is an isomorphism of real Lie groups

$$
G(\gamma)_{\infty} \stackrel{\simeq}{\longrightarrow} \operatorname{Sp}_{n}(\mathbb{R})^{s} \times \prod_{v \in \operatorname{Ram}_{\infty}(D)} \operatorname{Sp}\left(p_{v}, q_{v}\right)
$$

Here $s$ denotes the number of real places of $F$ which split $D$. Note that $G(\gamma)_{\infty}$ is connected and semisimple. The real Lie algebra $\mathfrak{g}(\gamma)_{\infty}$ of $G(\gamma)_{\infty}$ is isomorphic to

$$
\mathfrak{g}(\gamma)_{\infty} \cong \mathfrak{s p}(n, \mathbb{R})^{s} \oplus \bigoplus_{v \in \operatorname{Ram}_{\infty}(D)} \mathfrak{s p}\left(p_{v}, q_{v}\right)
$$

Recall that every maximal compact subgroup of the real Lie group $\operatorname{Sp}_{n}(\mathbb{R})$ is isomorphic to the unitary group $\mathrm{U}(n)$.

Consider the group $\operatorname{Sp}(n):=\operatorname{Sp}(n, 0)$. One can check that this is a compact connected semisimple real Lie group [Knapp 2002, page 111]. Moreover, it is a maximal compact subgroup of the special linear group $\mathrm{SL}_{n}(\mathbb{H})$.

Let $p, q \geq 0$ be integers with $p+q=n$. The Lie group $\operatorname{Sp}(p, q)$ is connected and semisimple [Knapp 2002, Proposition 1.145], and the compact subgroup $\operatorname{Sp}(p) \times \operatorname{Sp}(q)$ is a maximal compact subgroup. Given any maximal compact subgroup $K(\gamma)_{\infty} \subseteq G(\gamma)_{\infty}$, we obtain an isomorphism of Lie groups

$$
K(\gamma)_{\infty} \stackrel{\simeq}{\longrightarrow} \mathrm{U}(n)^{s} \times \prod_{v \in \operatorname{Ram}_{\infty}(D)} \operatorname{Sp}\left(p_{v}\right) \times \operatorname{Sp}\left(q_{v}\right) .
$$

5C2. The symmetric space. Consider the associated Riemannian symmetric space $X(\gamma)$ defined as $X(\gamma):=K(\gamma)_{\infty} \backslash G(\gamma)_{\infty}$. We have $\operatorname{dim} G(\gamma)=n(2 n+1)$, thus

$$
\operatorname{dim} G(\gamma)_{\infty}=n(2 n+1)[F: \mathbb{Q}] .
$$

The dimension of the unitary group $\mathrm{U}(n)$ is $n^{2}$ and consequently

$$
\operatorname{dim} K(\gamma)_{\infty}=s n^{2}+\sum_{v \in \operatorname{Ram}_{\infty}(D)} p_{v}\left(2 p_{v}+1\right)+q_{v}\left(2 q_{v}+1\right) .
$$

Subtraction of both dimensions yields an obviously even number:

$$
\operatorname{dim} X(\gamma)=\operatorname{sn}(n+1)+\sum_{v \in \operatorname{Ram}_{\infty}(D)} 4 p_{v} q_{v} .
$$


5C3. Lie algebras and complexifications. We complexify the Lie algebra $\mathfrak{g}(\gamma)_{\infty}$ and we obtain an isomorphism

$$
\mathfrak{g}(\gamma)_{\infty} \otimes_{\mathbb{R}} \mathbb{C} \cong \mathfrak{s p}(n, \mathbb{C})^{[F: \mathbb{Q}]} .
$$

The rank of this complex semisimple Lie algebra is $\operatorname{rk}\left(\mathfrak{g}(\gamma)_{\infty, \mathbb{C}}\right)=n[F: \mathbb{Q}]$. Let $\mathfrak{k}(\gamma)_{\infty}$ denote the Lie algebra of the maximal compact subgroup $K(\gamma)_{\infty}$. The complexification of this Lie algebra is isomorphic to

$$
\mathfrak{k}(\gamma)_{\infty} \otimes_{\mathbb{R}} \mathbb{C} \cong \mathfrak{g l}(n, \mathbb{C})^{s} \oplus \bigoplus_{v \in \operatorname{Ram}_{\infty}(D)} \mathfrak{s p}\left(p_{v}, \mathbb{C}\right) \oplus \mathfrak{s p}\left(q_{v}, \mathbb{C}\right) .
$$

The rank of $\mathfrak{k}(\gamma)_{\infty, \mathbb{C}}$ is $s n+\sum_{v \in \operatorname{Ram}_{\infty}(D)} p_{v}+q_{v}=n[F: \mathbb{Q}]$. Thus the complexified Lie algebras $\mathfrak{g}(\gamma)_{\infty, \mathbb{C}}$ and $\mathfrak{k}(\gamma)_{\infty, \mathbb{C}}$ have equal rank. The Weyl groups of these complex reductive Lie algebras are well known; in particular,

$$
\left|W\left(\mathfrak{g}(\gamma)_{\infty, \mathbb{C}}\right)\right|=\left(2^{n} n !\right)^{[F: \mathbb{Q}]}
$$

and

$$
\left|W\left(\mathfrak{k}(\gamma)_{\infty, \mathbb{C}}\right)\right|=(n !)^{s} \prod_{v \in \operatorname{Ram}_{\infty}(D)} 2^{p_{v}} p_{v} ! \cdot 2^{q_{v}} q_{v} !,
$$

as can be found in [Humphreys 1972, page 66]. The quotient of the cardinalities of the two Weyl groups is given by

$$
\frac{\left|W\left(\mathfrak{g}(\gamma)_{\infty, \mathbb{C}}\right)\right|}{\left|W\left(\mathfrak{k}(\gamma)_{\infty, \mathbb{C}}\right)\right|}=2^{n s} \prod_{v \in \operatorname{Ram}_{\infty}(D)}\left(\begin{array}{c}
n \\
p_{v}
\end{array}\right) .
$$

Remark. The linear algebraic $F$-group $G(\gamma) \times_{0} F$ is an inner form of the symplectic group $\mathrm{Sp}_{n}$; in particular it is a semisimple and simply connected group. Further this implies that the Tamagawa number $\tau(G(\gamma))$ is equal to one [Kottwitz 1988].

5C4. The metric form $B$. Recall that the Lie algebra of $G(\gamma)$ is a functor $\operatorname{Lie}(G(\gamma))$ which assigns to a commutative 0 -algebra $C$ the $C$-Lie algebra

$$
\operatorname{Lie}(G(\gamma))(C)=\left\{x \in\left(\Lambda \otimes_{\mathcal{O}} C\right)^{\times} \mid(\tau \mid \gamma)(x)=-x\right\} .
$$

For simplicity we write $\mathfrak{g}(\gamma)_{C}$ instead of $\operatorname{Lie}(G(\gamma))(C)$.

Consider the nondegenerate form $B: \mathfrak{g}(\gamma)_{F} \times \mathfrak{g}(\gamma)_{F} \rightarrow F$ defined by $B(x, y):=$ $-\frac{1}{2} \operatorname{trd}_{A}(x y)$. Let $\iota: F \rightarrow \mathbb{C}$ be an embedding of $F$ into the field of complex numbers. The central simple algebra $A=M_{n}(D)$ splits over $\mathbb{C}$ and we can choose a splitting $A \rightarrow M_{2 n}(\mathbb{C})$ such that $\tau \mid \gamma$ is the standard symplectic involution. Via this splitting the Lie algebra $\mathfrak{g}(\gamma)_{\mathbb{C}}$ is isomorphic to the complex semisimple Lie algebra $\mathfrak{s p}(n, \mathbb{C})$.

Proposition 5.15. Consider the compact Lie group $\mathrm{Sp}(n)$ and its Lie algebra

$$
\mathfrak{s p}(n):=\left\{x \in M_{n}(\mathbb{H}) \mid \bar{x}^{T}+x=0\right\},
$$


and the positive definite $\mathbb{R}$-bilinear form $B(x, y):=-\frac{1}{2} \operatorname{trd}(x y)$ on $\mathfrak{s p}(n)$. With respect to the right-invariant Riemann metric induced by $B$, the group $\operatorname{Sp}(n)$ has the volume

$$
\operatorname{vol}_{B}(\operatorname{Sp}(n))=\prod_{j=1}^{n} \frac{(2 \pi)^{2 j}}{2 \cdot(2 j-1) !} .
$$

Proof. The $\mathbb{C}$-linear extension of $B$ to $\mathfrak{s p}(n, \mathbb{C})$ is given by $B(x, y)=-\frac{1}{2} \operatorname{Tr}(x y)$. Recall that the Killing form $\beta$ on $\mathfrak{s p}(n, \mathbb{C})$ is the form $\beta(x, y)=(2 n+2) \operatorname{Tr}(x y)$ [Helgason 1978, Chapter III, §8] and hence $\beta=-4(n+1) B$. We conclude

$$
\operatorname{vol}_{\beta}(\operatorname{Sp}(n))=(4(n+1))^{\frac{n(2 n+1)}{2}} \operatorname{vol}_{B}(\operatorname{Sp}(n)) .
$$

The assertion follows from Ono's formula for the volume of a compact Lie group with respect to the Killing form [Ono 1966, Equation 3.4.9], which yields

$$
\operatorname{vol}_{\beta}(\operatorname{Sp}(n))=(4(n+1))^{\frac{n(2 n+1)}{2}} \prod_{j=1}^{n} \frac{(2 \pi)^{2 j}}{2 \cdot(2 j-1) !} .
$$

5C5. The modulus factor. Consider the $F$-bilinear form $B: \mathfrak{g}(\gamma)_{F} \times \mathfrak{g}(\gamma)_{F} \rightarrow F$ defined by $B(x, y):=-\frac{1}{2} \operatorname{trd}_{A}(x y)$. In this paragraph we will calculate the global modulus factor $m(B)=\prod_{v \in V_{f}} m(B)_{v}$ (see Section 3B2). Note that $\Lambda$ is in general not a free 0 -module, therefore we have to work locally.

We start with the finite places $v \in V_{f}$ where $D$ splits. The main observation is this: we can assume that $\Lambda \otimes_{\mathcal{O}} \mathrm{O}_{v}=M_{2 n}\left(\mathrm{O}_{v}\right)$ and that $\tau \mid \gamma$ is the standard symplectic involution. This follows from the next lemma.

Lemma 5.16. Let $R$ be a complete discrete valuation ring with field of fractions $k$ of characteristic char $(k) \neq 2$. Let $\sigma$ be an involution of symplectic type on $M_{2 n}(k)$ and let $\Lambda \subseteq M_{2 n}(k)$ be a maximal $R$-order which is $\sigma$-stable.

There is an element $g \in \mathrm{GL}_{2 n}(k)$ such that

(i) $g \Lambda g^{-1}=M_{2 n}(R)$, and

(ii) $g \sigma(x) g^{-1}=J\left(g x g^{-1}\right)^{T} J^{-1}$, where $J$ is the standard symplectic matrix.

Proof. It follows from [Reiner 2003, Theorem (17.3)] that there is an invertible matrix $a \in \mathrm{GL}_{2 n}(k)$ such that $a \Lambda a^{-1}=M_{2 n}(R)$. Moreover, $\sigma$ is an involution of symplectic type and we can consider $\operatorname{int}(a): M_{2 n}(k) \rightarrow M_{2 n}(k)$ as a splitting of the central simple $k$-algebra $M_{2 n}(k)$. There is a matrix $h \in \mathrm{GL}_{2 n}(k)$ such that $h^{T}=-h$ and $\operatorname{int}(a)(\sigma(x))=h(\operatorname{int}(a)(x))^{T} h^{-1}$ for every $x \in M_{2 n}(k)$.

Because $\Lambda$ is $\sigma$-stable, $h M_{2 n}(R) h^{-1}=M_{2 n}(R)$. After multiplication with some power of the prime element in $R$, we can assume $h \in \mathrm{GL}_{2 n}(R)$. On a free module over a complete discrete valuation ring, there is only one regular symplectic form up to isogeny (since $\operatorname{char}(k) \neq 2$ ); this means that there is $b \in \mathrm{GL}_{2 n}(R)$ such that 
$b h b^{T}=J$. Finally, we define $g:=b a$ and observe that $g \sigma(x) g^{-1}=b h\left(a x a^{-1}\right)^{T} h^{-1} b^{-1}=J\left(b^{-1}\right)^{T}\left(a x a^{-1}\right)^{T} b^{T} J^{-1}=J\left(g x g^{-1}\right)^{T} J^{-1}$ for every $x \in M_{2 n}(k)$.

Corollary 5.17. Let $\gamma \in Z^{1}\left(\tau^{*}, G(O)\right)$ be a cocycle and let $v \in V_{f}$ be a finite place of $F$ which splits $D$. There is an isomorphism of group schemes over $\mathbb{O}_{v}$ :

$$
G(\gamma) \times{ }_{0} \mathrm{O}_{v} \stackrel{\simeq}{\longrightarrow} \mathrm{Sp}_{n} \times \mathbb{Z} \mathrm{O}_{v}
$$

Proof. This follows directly from the previous lemma since $\tau \mid \gamma$ is an involution of symplectic type.

Proposition 5.18. Let $v \in V_{f}$ be a finite place which splits $D$. Consider the bilinear form $B: \mathfrak{g}(\gamma)_{F} \times \mathfrak{g}(\gamma)_{F} \rightarrow F$ defined by $B(x, y)=-\frac{1}{2} \operatorname{trd}(x y)$. The local modulus factor (see Section 3B2) is

$$
m(B)_{v}=|2|_{v}^{-n} .
$$

Proof. By Corollary 5.17 we can assume $G(\gamma)=\mathrm{Sp}_{n}$ over $\mathrm{O}_{v}$. This means

$$
\mathfrak{g}(\gamma)_{\mathscr{O}_{v}}=\mathfrak{s p}\left(n, \mathscr{O}_{v}\right)=\left\{x \in M_{2 n}\left(\mathscr{O}_{v}\right) \mid x^{T} J+J x=0\right\} .
$$

Note that the form $B$ is given by the analogous formula $B(x, y)=-\frac{1}{2} \operatorname{Tr}(x y)$. Recall that the elements of $\mathfrak{s p}\left(n, \mathcal{O}_{v}\right)$ are matrices of the form

$$
\left(\begin{array}{cc}
a & b \\
c & -a^{T}
\end{array}\right)
$$

with $a, b, c \in M_{n}\left(\mathcal{O}_{v}\right)$, where $b$ and $c$ are symmetric matrices. Let $E_{s, t}$ denote the elementary $2 n \times 2 n$ matrix with exactly one entry 1 in position $(s, t)$. We choose an $\mathrm{O}_{v}$-basis of $\mathfrak{s p}\left(n, \mathrm{O}_{v}\right)$ which is made up of the following elements:

(i) $a_{i, j}:=E_{i, j}-E_{j+n, i+n}$ for all $i, j \in\{1, \ldots, n\}$,

(ii) $b_{i, j}:=E_{i, j+n}+E_{j, i+n}$ for all $1 \leq i<j \leq n$,

(iii) $c_{i, j}:=E_{i+n, j}+E_{j+n, i}$ for all $1 \leq i<j \leq n$, and

(iv) $b_{i}:=E_{i, i+n}$ and $c_{i}:=E_{i+n, i}$ for all $i \in\{1, \ldots, n\}$.

We evaluate the form $B$ on all the basis vectors.

It is easy to observe that

$$
\begin{aligned}
0 & =B\left(a_{i, j}, c_{k, l}\right)=B\left(a_{i, j}, b_{k, l}\right)=B\left(a_{i, j}, b_{k}\right)=B\left(a_{i, j}, c_{k}\right) \\
& =B\left(c_{i, j}, c_{k, l}\right)=B\left(b_{i, j}, b_{k, l}\right)=B\left(c_{i}, c_{j}\right)=B\left(b_{i}, b_{j}\right) .
\end{aligned}
$$

for all $i, j, k, l$. Moreover, one readily verifies that $B\left(b_{i, j}, c_{k}\right)=B\left(c_{i, j}, b_{k}\right)=0$ for all $i, j, k$. The remaining cases yield

- $B\left(a_{i, j}, a_{k, l}\right)=-\delta_{j, k} \delta_{i, l}$ for all $i, j, k, l \in\{1, \ldots, n\}$, 
- $B\left(b_{i, j}, c_{k, l}\right)=-\delta_{i, k} \delta_{j, l}$ for all $i<j \leq n$ and $k<l \leq n$, and

- $B\left(b_{i}, c_{j}\right)=-\frac{1}{2} \delta_{i, j}$ for all $i, j \in\{1, \ldots, n\}$.

Using these results, we are able to calculate the modulus factor and obtain

$$
m(B)_{v}=\left|\operatorname{det}\left(\begin{array}{rr}
0 & -\frac{1}{2} \\
-\frac{1}{2} & 0
\end{array}\right)\right|_{v}^{n / 2}=|2|_{v}^{-n} .
$$

Proposition 5.19. Let $v \in \operatorname{Ram}_{f}(D)$ be a finite ramified place and $\mathfrak{p} \subset \mathcal{O}$ be the associated prime ideal. The local modulus factor for the group $G(\gamma)$ and the form $B$ defined in Section $5 C 4$ is

$$
m(B)_{v}=|2|_{v}^{-n} \mathrm{~N}(\mathfrak{p})^{-n(n+1) / 2} .
$$

Proof. The $F_{v}$-algebra $D_{v}:=D \otimes_{F} F_{v}$ is the unique quaternion division algebra over $F_{v}$ and $\Delta:=\Lambda_{D} \otimes_{\mathcal{O}} O_{v}$ is the unique maximal order in $D_{v}$.

Set $H:=G(1) \times O_{v}$. Due to Corollary 5.13 we can assume that $\gamma=1$, that is, $G(\gamma) \times \mathscr{O}_{v}$ is isomorphic to $H$. We define

$$
\mathfrak{h}:=\operatorname{Lie}(H)\left(\mathscr{O}_{v}\right)=\left\{x \in M_{n}(\Delta) \mid \tau(x)=-x\right\} .
$$

Recall that $\tau(x)=\bar{x}^{T}$.

Take an $\mathcal{O}_{v}$-basis $v_{0}, v_{1}, v_{2}, v_{3}$ of $\Delta$ such that $\operatorname{trd}_{D}\left(v_{0}\right)=1$ and $\operatorname{trd}_{D}\left(v_{i}\right)=0$ for $i=1,2,3$. Such a basis exists since $\operatorname{trd}_{D}: \Delta \rightarrow \mathcal{O}_{v}$ is surjective (maximal orders are smooth; see Proposition 2.4). We construct an $\mathrm{O}_{v}$-basis of the Lie algebra $\mathfrak{h}$, consisting of the following elements:

(i) $a_{s, i}:=v_{s} E_{i, i}$ for all $s \in\{1,2,3\}$ and $i \in\{1, \ldots, n\}$, and

(ii) $b_{s, i, j}:=v_{s} E_{i, j}-\bar{v}_{s} E_{j, i}$ for all $s \in\{0,1,2,3\}$ and $i, j \in\{1, \ldots, n\}$ with $i<j$.

We calculate the form $B$ on all basis vectors. Observe that $B\left(a_{s, i}, b_{t, k, l}\right)=0$ for all $s, t, i, k, l$. Moreover, for $s, t \in\{1,2,3\}$ and $i, j \in\{1, \ldots, n\}$ we find

$$
B\left(a_{s, i}, a_{t, j}\right)=-\frac{1}{2} \operatorname{trd}\left(v_{s} E_{i, i} v_{t} E_{j, j}\right)=-\frac{1}{2} \delta_{i, j} \operatorname{trd}_{D}\left(v_{s} v_{t}\right) .
$$

Finally, let $s, t \in\{0,1,2,3\}$ and let $i, j, k, l \in\{1, \ldots, n\}$ with $i<j$ and $k<l$. We obtain

$$
B\left(b_{s, i, j}, b_{t, k, l}\right)=\frac{1}{2} \operatorname{trd}_{D}\left(\bar{v}_{s} v_{t}+v_{s} \bar{v}_{t}\right) \delta_{i, k} \delta_{j, l}=\operatorname{trd}_{D}\left(v_{s} \bar{v}_{t}\right) \delta_{i, k} \delta_{j, l} .
$$

Summing up we obtain a formula for the modulus factor

$$
m(B)_{v}^{2}=\left|\frac{1}{8} \operatorname{det}\left(\operatorname{trd}\left(v_{s} v_{t}\right)\right)_{s, t=1,2,3}\right|_{v}^{n} \cdot\left|\operatorname{det}\left(\operatorname{trd}\left(v_{s} \bar{v}_{t}\right)\right)_{s, t=0,1,2,3}\right|_{v}^{n(n-1) / 2}
$$

Since the elements $\bar{v}_{0}, \bar{v}_{1}, \bar{v}_{2}, \bar{v}_{3}$ form an $\mathcal{O}_{v}$-basis of $\Delta$ as well, we see that the second term $\left|\operatorname{det}\left(\operatorname{trd}\left(v_{s} \bar{v}_{t}\right)\right)_{s, t=0,1,2,3}\right|_{v}$ is the valuation of the discriminant of $\Delta$. It is known that the discriminant of $\Delta$ is $\mathfrak{p}_{v}^{2}$ [Reiner 2003, Theorem (14.9)]. 
To calculate the first term in (3) we consider $w_{0}:=1$ and we define $w_{s}=v_{s}$ for $s=1,2,3$. Note that $w_{0}, w_{1}, w_{2}, w_{3}$ is in general not an $O_{v}$-basis of $\Delta$ since $\operatorname{trd}_{D}(1)=2$ need not be a unit in $\mathrm{O}_{v}$. We can write

$$
w_{0}=1=r_{0} v_{0}+r_{1} v_{1}+r_{2} v_{2}+r_{3} v_{3}
$$

for certain $r_{0}, r_{1}, r_{2}, r_{3}$ in $O_{v}$. Applying the reduced trace we get $2=\operatorname{trd}_{D}(1)=r_{0}$. Furthermore, this implies that the matrix $\left(\operatorname{trd}\left(w_{s} w_{t}\right)\right)_{s, t=0,1,2,3}$ can be written as a product of matrices

$$
\left(\begin{array}{cccc}
2 & r_{1} & r_{2} & r_{3} \\
0 & 1 & 0 & 0 \\
0 & 0 & 1 & 0 \\
0 & 0 & 0 & 1
\end{array}\right)\left(\operatorname{trd}\left(v_{i} v_{j}\right)\right)_{i, j=0,1,2,3}\left(\begin{array}{cccc}
2 & 0 & 0 & 0 \\
r_{1} & 1 & 0 & 0 \\
r_{2} & 0 & 1 & 0 \\
r_{3} & 0 & 0 & 1
\end{array}\right)
$$

Note that

$$
\left(\operatorname{trd}\left(w_{s} w_{t}\right)\right)_{s, t=0,1,2,3}=\left(\begin{array}{cccc}
2 & 0 & 0 & 0 \\
0 & \operatorname{trd}\left(v_{1} v_{1}\right) & \operatorname{trd}\left(v_{1} v_{2}\right) & \operatorname{trd}\left(v_{1} v_{3}\right) \\
0 & \operatorname{trd}\left(v_{2} v_{1}\right) & \operatorname{trd}\left(v_{2} v_{2}\right) & \operatorname{trd}\left(v_{2} v_{3}\right) \\
0 & \operatorname{trd}\left(v_{3} v_{1}\right) & \operatorname{trd}\left(v_{3} v_{2}\right) & \operatorname{trd}\left(v_{3} v_{3}\right)
\end{array}\right) .
$$

We deduce that $\left|\operatorname{det}\left(\operatorname{trd}\left(v_{s} v_{t}\right)\right)_{s, t=1,2,3}\right|_{v}=|2|_{v} \mathrm{~N}(\mathfrak{p})^{-2}$. In total the local modulus factor is

$$
m(B)_{v}=|2|_{v}^{-n} \mathrm{~N}(\mathfrak{p})^{-n-n(n-1) / 2}=|2|_{v}^{-n} \mathrm{~N}(\mathfrak{p})^{-n(n+1) / 2} .
$$

Corollary 5.20. Let $\gamma \in Z^{1}\left(\tau^{*}, G(0)\right)$ be a cocycle. The global modulus factor $m(B)$ for the group $G(\gamma)$ with respect to the form B defined in Section $5 C 4$ is

$$
m(B)=2^{n[F: \mathbb{Q}]}(-1)^{r n(n+1) / 2} \Delta_{\mathrm{rd}}(D)^{-n(n+1) / 2},
$$

where $\Delta_{\mathrm{rd}}(D)$ denotes the signed reduced discriminant of $D$ (see the definition on page 390).

Proof. By Proposition 5.18, Proposition 5.19, and an application of the product formula we obtain

$$
m(B)=\prod_{v \in V_{f}}|2|_{v}^{-n} \prod_{\mathfrak{p} \in \operatorname{Ram}_{f}(D)} \mathrm{N}(\mathfrak{p})^{-n(n+1) / 2}=2^{n[F: \mathbb{Q}]} \prod_{\mathfrak{p} \in \operatorname{Ram}_{f}(D)} \mathrm{N}(\mathfrak{p})^{-n(n+1) / 2} .
$$

5C6. The Euler characteristic of the fixed point groups. Let $\gamma \in Z^{1}\left(\tau^{*}, G(\mathcal{O})\right)$ be a cocycle. We are now able to compute the Euler characteristic of torsion-free arithmetic subgroups of $G(\gamma)$. In Theorem 5.21 we give a precise formula for principal congruence subgroups. More general subgroups can be treated analogously. 
For Theorem 5.21 the number field $F$ need not be totally real. Let $\mathfrak{a} \subset \mathcal{O}$ be a proper ideal. For a finite place $v \in V_{f}$ we define $K_{v}(\gamma, \mathfrak{a})$ to be the kernel of the reduction morphism $G(\gamma)\left(\mathscr{O}_{v}\right) \rightarrow G(\gamma)\left(\mathcal{O}_{v} / \mathfrak{a} \mathscr{O}_{v}\right)$. Note that $K_{v}(\gamma, \mathfrak{a})=G(\gamma)\left(\mathbb{O}_{v}\right)$ for almost all places. The group

$$
K_{f}(\gamma, \mathfrak{a}):=\prod_{v \in V_{f}} K_{v}(\gamma, \mathfrak{a})
$$

is an open compact subgroup of the locally compact group $G(\gamma)\left(\mathbb{A}_{f}\right)$. This subgroup is given by a local datum $(U, \alpha)$ (see Section 3B3). Let $v \in V_{f}$ be a finite place and let $\mathfrak{p}$ be the associated prime ideal. Let $e=v_{\mathfrak{p}}(\mathfrak{a})$ be the exponent of $\mathfrak{p}$ in $\mathfrak{a}$. We have $\alpha_{v}=1$ and $U_{v}=G(\gamma)(\mathcal{O} / \mathfrak{p})$ if $e=0$, otherwise $\alpha_{v}=e$ and $U_{v}=\{1\} \subseteq G(\gamma)\left(\mathcal{O} / \mathfrak{p}^{e}\right)$.

Let $G(\gamma)_{\infty}=\prod_{v \in V_{\infty}} G(\gamma)\left(F_{v}\right)$ and let $K(\gamma)_{\infty} \subseteq G(\gamma)_{\infty}$ be a maximal compact subgroup. For every real ramified place $v \in \operatorname{Ram}_{\infty}(D)$ we denote the local signature of the class of $\gamma$ in $H^{1}\left(\tau^{*}, G\left(F_{v}\right)\right)$ by $\left(p_{v}, q_{v}\right)$ (see the definition on page 393).

Theorem 5.21. Assume that $G(\gamma)(F)$ acts freely on $K(\gamma)_{\infty} K_{f}(\gamma, \mathfrak{a}) \backslash G(\gamma)(\mathbb{A})$. The Euler characteristic of the double quotient space

$$
S(\mathfrak{a}):=K(\gamma)_{\infty} K_{f}(\gamma, \mathfrak{a}) \backslash G(\gamma)(\mathbb{A}) / G(\gamma)(F)
$$

is nonzero if and only if $F$ is totally real. In this case the following formula holds:

$$
\chi(S(\mathfrak{a}))=2^{-n r} \mathrm{~N}(\mathfrak{a})^{n(2 n+1)} \Delta_{\mathrm{rd}}(D)^{n(n+1) / 2} \prod_{v \in \operatorname{Ram}_{\infty}(D)}\left(\begin{array}{c}
n \\
p_{v}
\end{array}\right) \prod_{j=1}^{n} M(j, \mathfrak{a}, D),
$$

where $M(j, \mathfrak{a}, D)$ is defined as

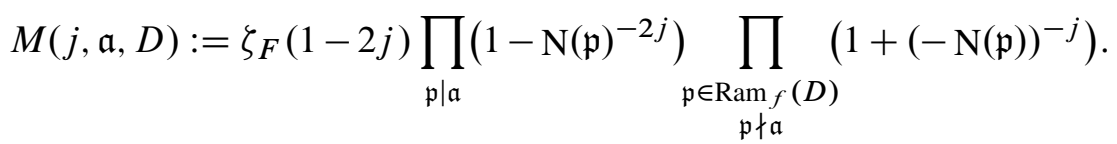

Here $r$ is the number of real places of $F$ where $D$ is ramified. The sign of $\chi(S(\mathfrak{a}))$ is $(-1)^{s n(n+1) / 2}$, where $s$ denotes the number of real places where $D$ splits.

Proof. It follows from the remark on page 384 that the Euler characteristic vanishes whenever $F$ has a complex place. Therefore we may assume that $F$ is totally real. We want to apply the adelic Euler characteristic formula (Theorem 3.3). We know that $G(\gamma)$ is a smooth group scheme over 0 (see Lemma 5.11). Further $G \times_{\mathcal{O}} F$ is an inner form of the symplectic group, and is thus a semisimple and simply connected algebraic group of dimension $d=n(2 n+1)$ (see the remark on page 401). Note further that by assumption $G(\gamma)(F)$ acts freely on $K(\gamma)_{\infty} K_{f}(\gamma, \mathfrak{a}) \backslash G(\gamma)(\mathbb{A})$.

Moreover, we observe that $\operatorname{dim} X(\gamma)$ is even (see Section 5C2) and that the complexified Lie algebras $\mathfrak{k}(\gamma)_{\infty} \otimes \mathbb{C}$ and $\mathfrak{g}(\gamma)_{\infty, \mathbb{C}}$ have equal rank (see Section 5C3). We conclude that the Euler characteristic does not vanish and Theorem 3.3 applies. 
We fix the nondegenerate bilinear form $B: \mathfrak{g}(\gamma)_{F} \times \mathfrak{g}(\gamma)_{F} \rightarrow F$ defined, as above, by $B(x, y):=-\frac{1}{2} \operatorname{trd}_{A}(x y)$. It is easy to see that the compact dual group $G(\gamma)_{u}$ of $G(\gamma)_{\infty}$ is isomorphic to $\operatorname{Sp}(n)^{[F: \mathbb{Q}]}$. Note further that $B$ is given by the same formula on each factor of the compact dual group. Therefore the volume is

$$
\operatorname{vol}_{B}\left(G(\gamma)_{u}\right)=\left(\prod_{j=1}^{n} \frac{(2 \pi)^{2 j}}{2 \cdot(2 j-1) !}\right)^{[F: \mathbb{Q}]}
$$

according to Proposition 5.15. Using the global modulus factor, calculated in Corollary 5.20, and the quotient of the orders of the involved Weyl groups, derived in Section 5C3, the adelic formula yields

$$
\begin{aligned}
\chi(S(\mathfrak{a}))= & (-1)^{[F: \mathbb{Q}] n(n+1) / 2}\left|d_{F}\right|^{d / 2} 2^{n s} \prod_{v \in \operatorname{Ram}_{\infty}(D)}\left(\begin{array}{c}
n \\
p_{v}
\end{array}\right) \\
& \cdot\left(\prod_{j=1}^{n} \frac{2 \cdot(2 j-1) !}{(2 \pi)^{2 j}}\right)^{[F: \mathbb{Q}]} 2^{-n[F: \mathbb{Q}]} \Delta_{\mathrm{rd}}(D)^{n(n+1) / 2} \prod_{\mathfrak{p} \in V_{f}} \frac{\mathrm{N}(\mathfrak{p})^{d \alpha_{\mathfrak{p}}}}{\left|U_{\mathfrak{p}}\right|} .
\end{aligned}
$$

Here $s$ denotes the number of real places of $F$ which split $D$. The only terms that can be negative are $(-1)^{[F: \mathbb{Q}] n(n+1) / 2}$ and the signed reduced discriminant. Consequently, the sign of the Euler characteristic is $(-1)^{s n(n+1) / 2}$.

Let $v \in V_{f}$ be a finite place with associated prime ideal $\mathfrak{p}$ and consider $\frac{\mathrm{N}(\mathfrak{p})^{d \alpha_{\mathfrak{p}}}}{\left|U_{\mathfrak{p}}\right|}$.

Case (a): $D$ splits at $v$ and $\mathfrak{p}$ does not divide $\mathfrak{a}$. In this case $\alpha_{\mathfrak{p}}=1$ and $U_{\mathfrak{p}}=$ $G(\gamma)(\mathcal{O} / \mathfrak{p})$. Since $G(\gamma)$ is isomorphic to $\mathrm{Sp}_{n}$ over $\mathrm{O}_{v}$ (see Corollary 5.17), there is an isomorphism of finite groups $G(\gamma)(\mathbb{O} / \mathfrak{p}) \cong \operatorname{Sp}_{n}(\mathbb{O} / \mathfrak{p})$. From [Wilson 2009, Section 3.5] we deduce that

$$
|G(\gamma)(\mathcal{O} / \mathfrak{p})|=\mathrm{N}(\mathfrak{p})^{d} \prod_{j=1}^{n}\left(1-\mathrm{N}(\mathfrak{p})^{-2 j}\right) .
$$

Case (b): $D$ is ramified at $v$ and $\mathfrak{p}$ does not divide $\mathfrak{a}$. In this situation we have $\alpha_{\mathfrak{p}}=1$ and $U_{\mathfrak{p}}=G(\gamma)(\mathcal{O} / \mathfrak{p})$. Let $k=0 / \mathfrak{p}$ be the finite residue class field and let $\ell / k$ be the unique quadratic extension. It is an easy exercise to show that $G(\gamma)(0 / p)$ is isomorphic to a semidirect product $U(\ell / k) \ltimes \operatorname{Sym}_{n}(\ell)$, where $U(\ell / k)$ denotes the unitary group of the quadratic extension $\ell / k$ and $\operatorname{Sym}_{n}(\ell)$ denotes the abelian group of symmetric $(n \times n)$-matrices with entries in $\ell$. Therefore (using [Wilson 2009, Section 3.6]) we get

$$
|G(\gamma)(\mathcal{O} / \mathfrak{p})|=\mathrm{N}(\mathfrak{p})^{d} \prod_{j=1}^{n}\left(1-(-\mathrm{N}(\mathfrak{p}))^{-j}\right) .
$$


Case (c): $\mathfrak{p}$ divides $\mathfrak{a}$. In this case $\alpha_{v}=v_{\mathfrak{p}}(\mathfrak{a})$ and $\left|U_{\mathfrak{p}}\right|=1$. Consequently,

$$
\frac{\mathrm{N}(\mathfrak{p})^{d \alpha_{\mathfrak{p}}}}{\left|U_{\mathfrak{p}}\right|}=N(\mathfrak{p})^{d v_{\mathfrak{p}}(\mathfrak{a})}
$$

The product of these terms is

$$
\prod_{\mathfrak{p} \in V_{f}} \frac{\mathrm{N}(\mathfrak{p})^{d \alpha_{\mathfrak{p}}}}{\left|U_{\mathfrak{p}}\right|}=\mathrm{N}(\mathfrak{a})^{d} \prod_{j=1}^{n}\left(\zeta_{F}(2 j) \prod_{\mathfrak{p} \mid \mathfrak{a}}\left(1-\mathrm{N}(\mathfrak{p})^{-2 j}\right) \prod_{\substack{\mathfrak{p} \in \operatorname{Ram}_{\mathfrak{p} \nmid \mathfrak{a}}(D) \\ \mathfrak{p} \in}}\left(1+(-\mathrm{N}(\mathfrak{p}))^{-j}\right)\right) .
$$

Here $\zeta_{F}$ denotes the zeta function of the number field $F$.

Note that $d=n(2 n+1)=\sum_{j=1}^{n} 4 j-1$ and so $\left|d_{F}\right|^{d / 2}=\prod_{j=1}^{n}\left|d_{F}\right|^{(4 j-1) / 2}$. The functional equation of the zeta function of the totally real number field $F$ [Weil 1995, Chapter VII §6, Theorem 3] yields

$$
\zeta_{F}(2 j)\left|d_{F}\right|^{(4 j-1) / 2}\left(\frac{2 \cdot(2 j-1) !}{(2 \pi)^{2 j}}\right)^{[F: \mathbb{Q}]}=(-1)^{j[F: \mathbb{Q}]} \zeta_{F}(1-2 j)
$$

for every integer $j \geq 1$. Using this we see that

$\left|d_{F}\right|^{d / 2}\left(\prod_{j=1}^{n} \frac{2 \cdot(2 j-1) !}{(2 \pi)^{2 j}}\right)^{[F: \mathbb{Q}]} \prod_{j=1}^{n} \zeta_{F}(2 j)=(-1)^{[F: \mathbb{Q}] n(n+1) / 2} \prod_{j=1}^{n} \zeta_{F}(1-2 j)$.

Substitute this into (4); then a simple calculation proves the claim.

5D. Proof of the main theorem. The notation and assumptions are those of the introduction. As usual $F$ denotes an algebraic number field and $O$ denotes its ring of integers. Let $D$ be a quaternion algebra defined over $F$ and let $\Lambda_{D} \subseteq D$ be a maximal 0 -order. Let $n \geq 1$ be an integer; we consider the central simple $F$-algebra $A=M_{n}(D)$ and the maximal 0 -order $\Lambda=M_{n}\left(\Lambda_{D}\right)$. Further $G:=\mathrm{SL}_{\Lambda}$ is the smooth 0 -group scheme defined as the kernel of the reduced norm over the order $\Lambda$ (see the first definition on page 376).

We say that the quaternion algebra $D$ over $F$ is totally definite if $F$ is totally real and $D$ ramifies at every real place of $F$.

The algebraic group $G \times_{\mathbb{O}} F$ has strong approximation since it is an $F$-simple, simply connected group and $G_{\infty} \cong \mathrm{SL}_{2 n}(\mathbb{R})^{s} \times \mathrm{SL}_{n}(\mathbb{H})^{r} \times \mathrm{SL}_{2 n}(\mathbb{C})^{t}$ is not compact. Since the group $\mathrm{SL}_{1}(\mathbb{H})$ is compact, we need the assumption that $n \geq 2$ if $D$ is totally definite.

Let $K_{\infty} \subseteq G_{\infty}$ be a $\tau^{*}$-stable maximal compact subgroup. Further, let $K_{f}$ be the open compact subgroup of $G\left(\mathbb{A}_{f}\right)$, which satisfies $\Gamma(\mathfrak{a})=K_{f} \cap G(F)$ (see Section 5A3). Since $\Gamma(\mathfrak{a})$ is torsion-free and $\tau^{*}$-stable, we can apply Theorem 4.2 
and we obtain

$$
\mathscr{L}\left(\tau^{*}, \Gamma(\mathfrak{a}), W\right)=\sum_{\eta \in \mathscr{H}^{1}\left(\tau^{*}\right)} \chi\left(\vartheta^{-1}(\eta)\right) \operatorname{Tr}\left(\tau^{*} \mid W\left(\gamma_{\eta}\right)\right)
$$

Here $\gamma_{\eta}$ is any representative of the $H^{1}\left(\tau^{*}, G(F)\right)$ component of $\eta$ and

$$
\vartheta:\left(K_{\infty} K_{f} \backslash G(\mathbb{A}) / G(F)\right)^{\tau^{*}} \rightarrow \mathscr{H}^{1}\left(\tau^{*}\right)
$$

is the surjective continuous map defined in Section 4.

By Theorem 5.10 the projection $\pi: \mathscr{H}^{1}\left(\tau^{*}\right) \rightarrow H^{1}\left(\tau^{*}, G(F)\right)$ is injective and there is an exact sequence of pointed sets

$$
1 \longrightarrow \mathscr{H}^{1}\left(\tau^{*}\right) \stackrel{\pi}{\longrightarrow} H^{1}\left(\tau^{*}, G(F)\right) \stackrel{\mathrm{pf}_{\tau}}{\longrightarrow}\{ \pm 1\} \longrightarrow 1
$$

We deduce that, given a class $\eta \in \mathscr{H}^{1}\left(\tau^{*}\right)$, every representative $\gamma_{\eta} \in \pi(\eta)$ has pfaffian one, and hence they all describe the trivial class in $H^{1}\left(\tau^{*}, G(\bar{F})\right)$. Thus there is some $g \in G(\bar{F})$ such that $\gamma_{\eta}=g^{-1} \tau_{g}^{*}$. It follows that $\operatorname{Tr}\left(\tau^{*} \mid W\left(\gamma_{\eta}\right)\right)=\operatorname{Tr}\left(\tau^{*} \mid W\right)$ since $\tau^{*} \mid \gamma_{\eta}=\rho(g)^{-1} \circ \tau^{*} \circ \rho(g)$ on $W$.

As a next step we describe the fixed point components. Let $\eta \in \mathscr{H}^{1}\left(\tau^{*}\right)$. Using strong approximation we can choose representing cocycles $k_{\eta}$ in $Z^{1}\left(\tau^{*}, K_{\infty} K_{f}\right)$ and $\gamma_{\eta}$ in $Z^{1}\left(\tau^{*}, \Gamma(\mathfrak{a})\right)$, and an element $a_{\infty} \in G_{\infty}$ such that

$$
\eta=\left(\left[k_{\eta}\right],\left[\gamma_{\eta}\right]\right) \text { and } \tau^{*} a_{\infty}=k_{\eta}^{-1} a_{\infty} \gamma_{\eta}
$$

We write $k_{\eta}=k_{\infty} k_{0}$ with $k_{\infty} \in K_{\infty}$ and $k_{0} \in K_{f}$. Note that $k_{0}=\gamma_{\eta}$ considered as elements in $G\left(\mathbb{A}_{f}\right)$. By Lemma 4.1 there is a homeomorphism

$$
\vartheta^{-1}(\eta) \stackrel{\simeq}{\longrightarrow}\left(a_{\infty}^{-1} K_{\infty}^{\tau^{*} \mid k_{\infty}} a_{\infty}\right) K_{f}\left(\gamma_{\eta}, \mathfrak{a}\right) \backslash G\left(\gamma_{\eta}\right)(\mathbb{A}) / G\left(\gamma_{\eta}\right)(F)
$$

In fact $\left(a_{\infty}^{-1} K_{\infty}^{\tau^{*} \mid k_{\infty}} a_{\infty}\right)$ is a maximal compact subgroup of $G\left(\gamma_{\eta}\right)_{\infty}$.

Let $v \in \operatorname{Ram}_{\infty}(D)$ and let $\left(p_{v}, q_{v}\right)$ denote the local signature of $\gamma_{\eta}$ at $v$. By Theorem 5.21 the Euler characteristic of the fixed point component is zero if $F$ has a complex place. If $F$ is totally real, which we assume from now on, then

$$
\chi\left(\vartheta^{-1}(\eta)\right)=2^{-n r} \mathrm{~N}(\mathfrak{a})^{n(2 n+1)} \Delta_{\mathrm{rd}}(D)^{n(n+1) / 2} \prod_{v \in \operatorname{Ram}_{\infty}(D)}\left(\begin{array}{c}
n \\
p_{v}
\end{array}\right) \prod_{j=1}^{n} M(j, \mathfrak{a}, D)
$$

The short exact sequence (6), in combination with the Hasse principle and Lemma 5.9, shows that the map which takes cocycles to their local signatures induces a bijection

$$
\mathscr{H}^{1}\left(\tau^{*}\right) \stackrel{\simeq}{\longrightarrow} \prod_{v \in \operatorname{Ram}_{\infty}(D)}\left\{\left(p_{v}, q_{v}\right) \mid p_{v}+q_{v}=n \text { and } q_{v} \text { is even }\right\}
$$


The following identity can be easily verified:

$$
\sum_{\eta \in \mathscr{H}^{1}\left(\tau^{*}\right)} \prod_{v \in \operatorname{Ram}_{\infty}(D)}\left(\begin{array}{c}
n \\
q_{v}
\end{array}\right)=\sum_{u_{1}, \ldots, u_{r}=0}^{\left[\frac{n}{2}\right]} \prod_{i=1}^{r}\left(\begin{array}{c}
n \\
2 u_{i}
\end{array}\right)=2^{r(n-1)} .
$$

As a final step we substitute all results in formula (5) and observe:

$$
\mathscr{L}\left(\tau^{*}, \Gamma(\mathfrak{a}), W\right)=2^{-r} \mathrm{~N}(\mathfrak{a})^{n(2 n+1)} \Delta_{\mathrm{rd}}(D)^{n(n+1) / 2} \operatorname{Tr}\left(\tau^{*} \mid W\right) \prod_{j=1}^{n} M(j, \mathfrak{a}, D) .
$$

Note that the Lefschetz number is nonzero precisely when $F$ is totally real and $\operatorname{Tr}\left(\tau^{*} \mid W\right)$ does not vanish.

5E. The growth of the total Betti number. There are many recent results on the asymptotic behaviour of Betti numbers of arithmetic groups. Most of these results are upper bound results - a strong asymptotic upper bound was obtained by Calegari and Emerton [2009]. However, there are no strong lower bound results. It seems that the only available lower bound results are nonvanishing results for certain degrees in the cohomology. Indeed, there is a geometric method to construct cohomology classes in a given degree for cocompact arithmetic groups. This method originated from the work of Millson and Raghunathan [1981] and has been further elaborated by Rohlfs and Schwermer [1993]. Another result that can be interpreted as a result on lower bounds has been obtained by Venkataramana [2008]. In this last section we prove Corollary 1.1 to show that Lefschetz numbers provide asymptotic lower bounds for the total Betti number. The only remaining step is to relate the Lefschetz number to the index of the congruence subgroup $\Gamma(\mathfrak{a})$. Let $F$ be a totally real number field. If $D$ is totally definite we assume $n \geq 2$ such that $G=\mathrm{SL}_{\Lambda}$ has strong approximation.

Lemma 5.22. The index $[G(\mathcal{O}): \Gamma(\mathfrak{a})]$ of $\Gamma(\mathfrak{a})$ in $G(\mathcal{O})$ is

$$
\mathrm{N}(\mathfrak{a})^{4 n^{2}-1} \prod_{\substack{\mathfrak{p} \in \operatorname{Ram}_{f}(D) \\ \mathfrak{p} \nmid \mathfrak{a}}}\left(\prod_{j=2}^{2 n}\left(1-\mathrm{N}(\mathfrak{p})^{-j}\right)\right) \prod_{\substack{\mathfrak{p} \in \operatorname{Ram}_{f}(D) \\ \mathfrak{p} \mid \mathfrak{a}}}\left(\left(1+\mathrm{N}(\mathfrak{p})^{-1}\right) \prod_{j=2}^{n}\left(1-\mathrm{N}(\mathfrak{p})^{-2 j}\right)\right) .
$$

In particular, the term $[G(\mathcal{O}): \Gamma(\mathfrak{a})] N(\mathfrak{a})^{-4 n^{2}+1}$ is bounded from above and from below independent of $\mathfrak{a}$,

$$
\begin{aligned}
\prod_{j=2}^{2 n} \zeta_{F}(j)^{-1} & \leq[G(O): \Gamma(\mathfrak{a})] N(\mathfrak{a})^{-4 n^{2}+1} \\
& \leq \prod_{\mathfrak{p} \in \operatorname{Ram}_{f}(D)}\left(1+\mathrm{N}(\mathfrak{p})^{-1}\right) .
\end{aligned}
$$


Proof. Using the smoothness of the group scheme combined with strong approximation, there is a short exact sequence of groups

$$
1 \longrightarrow \Gamma(\mathfrak{a}) \longrightarrow G(\mathbb{O}) \longrightarrow G(\mathbb{O} / \mathfrak{a}) \longrightarrow 1,
$$

from which we deduce $[G(\mathcal{O}): \Gamma(\mathfrak{a})]=\prod_{\mathfrak{p} \mid \mathfrak{a}}\left|G\left(O_{\mathfrak{p}} / \mathfrak{a} O_{\mathfrak{p}}\right)\right|$. Let $\mathfrak{p}$ be a prime ideal which divides $\mathfrak{a}$, say $v_{\mathfrak{p}}(\mathfrak{a})=e \geq 1$. Then $\mathscr{O}_{\mathfrak{p}} / \mathfrak{a} \mathscr{O}_{\mathfrak{p}} \cong \mathscr{O}_{\mathfrak{p}} / \mathfrak{p}^{e} \mathscr{O}_{\mathfrak{p}}$ and it follows from the smoothness of $G$ that

$$
\left|G\left(\mathscr{O}_{\mathfrak{p}} / \mathfrak{p}^{e} \mathscr{O}_{\mathfrak{p}}\right)\right|=\mathrm{N}(\mathfrak{p})^{(e-1) d}\left|G\left(\mathscr{O}_{\mathfrak{p}} / \mathfrak{p} \mathscr{O}_{\mathfrak{p}}\right)\right|,
$$

where $d$ is the dimension of the group $G \times_{\mathbb{O}} F$ (use [Oesterlé 1984, Section I.2.1]). The dimension of $G$ is $d=4 n^{2}-1$.

If $\mathfrak{p} \in \operatorname{Ram}_{f}(D)$, then one can show that

$$
\left|G\left(\mathscr{O}_{\mathfrak{p}} / \mathfrak{p} \bigcirc_{\mathfrak{p}}\right)\right|=\mathrm{N}(\mathfrak{p})^{4 n^{2}-1}\left(1+\mathrm{N}(\mathfrak{p})^{-1}\right) \prod_{j=2}^{n}\left(1-\mathrm{N}(\mathfrak{p})^{-2 j}\right) .
$$

If otherwise $\mathfrak{p} \notin \operatorname{Ram}_{f}(D)$, then $G \times_{\mathscr{O}} \mathcal{O}_{\mathfrak{p}}$ is isomorphic to the special linear group $\mathrm{SL}_{2 n}$. We deduce that

$$
\left|G\left(\mathscr{O}_{\mathfrak{p}} / \mathfrak{p} \bigcirc_{\mathfrak{p}}\right)\right|=\mathrm{N}(\mathfrak{p})^{4 n^{2}-1} \prod_{j=2}^{2 n}\left(1-\mathrm{N}(\mathfrak{p})^{-j}\right),
$$

due to [Wilson 2009, Section 3.3.1]. Now the assertions can be readily verified.

Proof of Corollary 1.1. Since $\Gamma_{0}(\mathfrak{a})$ is a subgroup of finite index in $\Gamma(\mathfrak{a})$, we obtain from [Serre 1979, Chapter VII, Proposition 6] that $b_{i}(\Gamma(\mathfrak{a})) \leq b_{i}\left(\Gamma_{0}(\mathfrak{a})\right)$. It follows directly from the main theorem that there is a positive real number $b>0$, depending on $F, D$ and $n$, such that

$$
b \mathrm{~N}(\mathfrak{a})^{n(2 n+1)} \leq\left|\mathscr{L}\left(\tau^{*}, \Gamma(\mathfrak{a}), \mathbb{C}\right)\right|
$$

for every ideal $\mathfrak{a} \subseteq \mathcal{O}$ that makes $\Gamma(\mathfrak{a})$ torsion-free. Since $B(\Gamma(\mathfrak{a})) \geq\left|\mathscr{L}\left(\tau^{*}, \Gamma(\mathfrak{a}), \mathbb{C}\right)\right|$, it follows from Lemma 5.22 that

$$
B(\Gamma(\mathfrak{a})) \geq a[G(\mathcal{O}): \Gamma(\mathfrak{a})]^{\frac{n(2 n+1)}{4 n^{2}-1}}
$$

for some $a>0$ depending on $F, D$ and $n$. We obtain

$$
\begin{aligned}
B\left(\Gamma_{0}(\mathfrak{a})\right) & \geq a[G(\mathcal{O}): \Gamma(\mathfrak{a})]^{\frac{n(2 n+1)}{4 n^{2}-1}} \geq a\left[G(\mathcal{O}) \cap \Gamma_{0}: \Gamma_{0}(\mathfrak{a})\right]^{\frac{n(2 n+1)}{4 n^{2}-1}} \\
& =a\left(\left[\Gamma_{0}: G(\mathcal{O}) \cap \Gamma_{0}\right]^{-1}\left[\Gamma_{0}: \Gamma_{0}(\mathfrak{a})\right]\right)^{\frac{n(2 n+1)}{4 n^{2}-1}}
\end{aligned}
$$

We define $\kappa=a\left[\Gamma_{0}: G(\mathcal{O}) \cap \Gamma_{0}\right]^{-\frac{n(2 n+1)}{4 n^{2}-1}}$. 
Acknowledgements. I would like to thank Professor J. Schwermer for his support during my thesis work, upon which this article is based.

\section{References}

[An and Wang 2008] J. An and Z. Wang, "Nonabelian cohomology with coefficients in Lie groups", Trans. Amer. Math. Soc. 360:6 (2008), 3019-3040. MR 2009k:20116 Zbl 1194.22006

[Batyrev 1999] V. V. Batyrev, "Birational Calabi-Yau $n$-folds have equal Betti numbers", pp. 1-11 in New trends in algebraic geometry (Warwick, 1996), edited by K. Hulek et al., London Math. Soc. Lecture Note Ser. 264, Cambridge Univ. Press, 1999. MR 2000i:14059 Zbl 0955.14028

[Borel 1963] A. Borel, "Some finiteness properties of adele groups over number fields", Inst. Hautes Études Sci. Publ. Math. 16 (1963), 5-30. MR 34 \#2578 Zbl 0135.08902

[Borel and Serre 1973] A. Borel and J.-P. Serre, "Corners and arithmetic groups", Comment. Math. Helv. 48 (1973), 436-491. MR 52 \#8337 Zbl 0274.22011

[Bourbaki 1963] N. Bourbaki, Intégration. Chapitre 7: Mesure de Haar; Chapitre 8: Convolution et représentations, Actualités Scientifiques et Industrielles 1306, Hermann, Paris, 1963. MR 31 \#3539 Zbl 0156.03204

[Calegari and Emerton 2009] F. Calegari and M. Emerton, "Bounds for multiplicities of unitary representations of cohomological type in spaces of cusp forms", Ann. of Math. (2) 170:3 (2009), 1437-1446. MR 2011c:22032 Zbl 1195.22015

[Demazure and Gabriel 1970] M. Demazure and P. Gabriel, Groupes algébriques, I: Géométrie algébrique, généralités, groupes commutatifs, Masson, Paris, 1970. MR 46 \#1800 Zbl 0203.23401

[Fainsilber and Morales 1999] L. Fainsilber and J. Morales, "An injectivity result for Hermitian forms over local orders”, Illinois J. Math. 43:2 (1999), 391-402. MR 2000g:11026 Zbl 0939.11020

[Grothendieck 1964] A. Grothendieck, "Éléments de géométrie algébrique, IV: Étude locale des schémas et des morphismes de schémas, I”, Inst. Hautes Études Sci. Publ. Math. 20 (1964), 5-259. MR 30 \#3885 Zbl 0136.15901

[Harder 1971] G. Harder, "A Gauss-Bonnet formula for discrete arithmetically defined groups", Ann. Sci. École Norm. Sup. (4) 4 (1971), 409-455. MR 46 \#8255 Zbl 0232.20088

[Harder 1975] G. Harder, "On the cohomology of SL(2, O)”, pp. 139-150 in Lie groups and their representations (Budapest, 1971), edited by I. M. Gelfand, Halsted, New York, 1975. MR 54 \#12977 Zbl 0395.57028

[Helgason 1978] S. Helgason, Differential geometry, Lie groups, and symmetric spaces, Pure and Applied Mathematics 80, Academic Press, New York, 1978. MR 80k:53081 Zbl 0451.53038

[Humphreys 1972] J. E. Humphreys, Introduction to Lie algebras and representation theory, Graduate Texts in Mathematics 9, Springer, New York, 1972. MR 48 \#2197 Zbl 0254.17004

[Katok 1992] S. Katok, Fuchsian groups, University of Chicago Press, Chicago, IL, 1992. MR 93d: 20088 Zbl 0753.30001

[Kionke 2012] S. Kionke, Lefschetz numbers of involutions on arithmetic subgroups of inner forms of the special linear group, Ph.D. thesis, Universität Wien, Vienna, 2012.

[Kionke and Schwermer 2012] S. Kionke and J. Schwermer, "On the growth of the first Betti number of arithmetic hyperbolic 3-manifolds”, preprint, 2012. arXiv 1204.3750

[Klingen 1962] H. Klingen, "Über die Werte der Dedekindschen Zetafunktion”, Math. Ann. 145 (1962), 265-272. MR 24 \#A3138 Zbl 0101.03002 
[Knapp 2002] A. W. Knapp, Lie groups beyond an introduction, 2nd ed., Progress in Mathematics 140, Birkhäuser, Boston, MA, 2002. MR 2003c:22001 Zbl 1075.22501

[Knus et al. 1998] M.-A. Knus, A. Merkurjev, M. Rost, and J.-P. Tignol, The book of involutions, American Mathematical Society Colloquium Publications 44, Amer. Math. Soc., Providence, RI, 1998. MR 2000a:16031 Zbl 0955.16001

[Kottwitz 1988] R. E. Kottwitz, “Tamagawa numbers”, Ann. of Math. (2) 127:3 (1988), 629-646. MR 90e:11075 Zbl 0678.22012

[Krämer 1985] N. Krämer, Beiträge zur Arithmetik imaginärquadratischer Zahlkörper, Ph.D. thesis, Rheinische Friedrich-Wilhelms-Universität, Bonn, 1985.

[Lai 1991] K. F. Lai, "Lefschetz numbers and unitary groups", Bull. Austral. Math. Soc. 43:2 (1991), 193-209. MR 92a:11063 Zbl 0724.11019

[Lee and Schwermer 1983] R. Lee and J. Schwermer, "The Lefschetz number of an involution on the space of harmonic cusp forms of $\mathrm{SL}_{3}$ ”, Invent. Math. 73:2 (1983), 189-239. MR 84k:22016 Zbl 0525.10014

[Millson and Raghunathan 1981] J. J. Millson and M. S. Raghunathan, "Geometric construction of cohomology for arithmetic groups, I", Proc. Indian Acad. Sci. Math. Sci. 90:2 (1981), 103-123. MR 83d:22008 Zbl 0524.22012

[Oesterlé 1984] J. Oesterlé, "Nombres de Tamagawa et groupes unipotents en caractéristique $p$ ", Invent. Math. 78:1 (1984), 13-88. MR 86i:11016 Zbl 0542.20024

[Ono 1966] T. Ono, "On algebraic groups and discontinuous groups", Nagoya Math. J. 27 (1966), 279-322. MR 33 \#7342 Zbl 0166.29802

[Platonov and Rapinchuk 1994] V. Platonov and A. Rapinchuk, Algebraic groups and number theory, Pure and Applied Mathematics 139, Academic Press, Boston, MA, 1994. MR 95b:11039 Zbl 0841.20046

[Prasad 1989] G. Prasad, "Volumes of $S$-arithmetic quotients of semi-simple groups", Inst. Hautes Études Sci. Publ. Math. 69 (1989), 91-117. MR 91c:22023 Zbl 0695.22005

[Reiner 2003] I. Reiner, Maximal orders, London Mathematical Society Monographs (N.S.) 28, Oxford University Press, 2003. MR 2004c:16026 Zbl 1024.16008

[Rohlfs 1978] J. Rohlfs, “Arithmetisch definierte Gruppen mit Galoisoperation”, Invent. Math. 48:2 (1978), 185-205. MR 80j:20043 Zbl 0391.14007

[Rohlfs 1981] J. Rohlfs, "The Lefschetz number of an involution on the space of classes of positive definite quadratic forms", Comment. Math. Helv. 56:2 (1981), 272-296. MR 83a:10037 Zbl 0474.10019

[Rohlfs 1985] J. Rohlfs, "On the cuspidal cohomology of the Bianchi modular groups", Math. Z. 188:2 (1985), 253-269. MR 86e:11042 Zbl 0535.20028

[Rohlfs 1990] J. Rohlfs, "Lefschetz numbers for arithmetic groups", pp. 303-313 in Cohomology of arithmetic groups and automorphic forms (Luminy-Marseille, 1989), edited by J.-P. Labesse and J. Schwermer, Lecture Notes in Math. 1447, Springer, Berlin, 1990. MR 92d:11055 Zbl 0762.11023

[Rohlfs and Schwermer 1993] J. Rohlfs and J. Schwermer, "Intersection numbers of special cycles", J. Amer. Math. Soc. 6:3 (1993), 755-778. MR 94a:11075 Zbl 0811.11039

[Rohlfs and Schwermer 1998] J. Rohlfs and J. Schwermer, "An arithmetic formula for a topological invariant of Siegel modular varieties", Topology 37:1 (1998), 149-159. MR 98f:11044 Zbl 0926.11036

[Scharlau 1985] W. Scharlau, Quadratic and Hermitian forms, Grundlehren der Mathematischen Wissenschaften 270, Springer, Berlin, 1985. MR 86k:11022 Zbl 0584.10010 
[Sengün and Türkelli 2012] M. H. Sengün and S. Türkelli, "On the dimension of cohomology of Bianchi groups", preprint, 2012. arXiv 1204.0470v2

[Serre 1971] J.-P. Serre, "Cohomologie des groupes discrets", pp. 77-169 in Prospects in mathematics (Princeton, NJ, 1970), Ann. of Math. Studies 70, Princeton Univ. Press, 1971. MR 52 \#5876 Zbl 0235.22020

[Serre 1979] J.-P. Serre, Local fields, Graduate Texts in Mathematics 67, Springer, New York, 1979. MR 82e:12016 Zbl 0423.12016

[Serre 1994] J.-P. Serre, Cohomologie galoisienne, 5th ed., Lecture Notes in Mathematics 5, Springer, Berlin, 1994. MR 96b:12010 Zbl 0812.12002

[Shimura 1963] G. Shimura, "Arithmetic of alternating forms and quaternion hermitian forms", $J$. Math. Soc. Japan 15 (1963), 33-65. MR 26 \#3694 Zbl 0121.28102

[Shimura 1971] G. Shimura, Introduction to the arithmetic theory of automorphic functions, Publications of the Mathematical Society of Japan 11, Princeton University Press, 1971. MR 47 \#3318 Zbl 0221.10029

[Siegel 1969] C. L. Siegel, "Berechnung von Zetafunktionen an ganzzahligen Stellen", Nachr. Akad. Wiss. Göttingen Math.-Phys. Kl. II 1969 (1969), 87-102. MR 40 \#5570 Zbl 0186.08804

[Venkataramana 2008] T. N. Venkataramana, "Virtual Betti numbers of compact locally symmetric spaces”, Israel J. Math. 166 (2008), 235-238. MR 2010a:22015 Zbl 1221.57040

[Weil 1995] A. Weil, Basic number theory, 2nd ed., Springer, Berlin, 1995. MR 96c:11002 Zbl 0823.11001

[Wilson 2009] R. A. Wilson, The finite simple groups, Graduate Texts in Mathematics 251, Springer, London, 2009. MR 2011e:20018 Zbl 1203.20012

Received May 22, 2013.

STEFFEN KIONKE

MATHEMATISCHES INSTITUT

HEINRICH-HEINE-UNIVERSITÄT DÜSSELDORF

UNIVERSITÄTSSTR. 1

40225 DÜSSELDORF

GERMANY

steffen.kionke@uni-duesseldorf.de 


\title{
PACIFIC JOURNAL OF MATHEMATICS
}

\author{
msp.org/pjm
}

Founded in 1951 by E. F. Beckenbach (1906-1982) and F. Wolf (1904-1989)

\section{EDITORS}

Don Blasius (Managing Editor)

Department of Mathematics

University of California

Los Angeles, CA 90095-1555

blasius@math.ucla.edu

\author{
Paul Balmer \\ Department of Mathematics \\ University of California \\ Los Angeles, CA 90095-1555 \\ balmer@math.ucla.edu \\ Robert Finn \\ Department of Mathematics \\ Stanford University \\ Stanford, CA 94305-2125 \\ finn@math.stanford.edu \\ Sorin Popa \\ Department of Mathematics \\ University of California \\ Los Angeles, CA 90095-1555 \\ popa@math.ucla.edu
}

\author{
Vyjayanthi Chari \\ Department of Mathematics \\ University of California \\ Riverside, CA 92521-0135 \\ chari@math.ucr.edu \\ Kefeng Liu \\ Department of Mathematics \\ University of California \\ Los Angeles, CA 90095-1555 \\ liu@math.ucla.edu \\ Jie Qing \\ Department of Mathematics \\ University of California \\ Santa Cruz, CA 95064 \\ qing@ cats.ucsc.edu
}

\section{PRODUCTION}

Silvio Levy, Scientific Editor, production@msp.org

\section{SUPPORTING INSTITUTIONS}

ACADEMIA SINICA, TAIPEI

CALIFORNIA INST. OF TECHNOLOGY

INST. DE MATEMÁTICA PURA E APLICADA

KEIO UNIVERSITY

MATH. SCIENCES RESEARCH INSTITUTE

NEW MEXICO STATE UNIV.

OREGON STATE UNIV.

\author{
STANFORD UNIVERSITY \\ UNIV. OF BRITISH COLUMBIA \\ UNIV. OF CALIFORNIA, BERKELEY \\ UNIV. OF CALIFORNIA, DAVIS \\ UNIV. OF CALIFORNIA, LOS ANGELES \\ UNIV. OF CALIFORNIA, RIVERSIDE \\ UNIV. OF CALIFORNIA, SAN DIEGO \\ UNIV. OF CALIF., SANTA BARBARA
}

\author{
Daryl Cooper \\ Department of Mathematics \\ University of California \\ Santa Barbara, CA 93106-3080 \\ cooper@math.ucsb.edu \\ Jiang-Hua Lu \\ Department of Mathematics \\ The University of Hong Kong \\ Pokfulam Rd., Hong Kong \\ jhlu@maths.hku.hk \\ Paul Yang \\ Department of Mathematics \\ Princeton University \\ Princeton NJ 08544-1000 \\ yang@math.princeton.edu
}

These supporting institutions contribute to the cost of publication of this Journal, but they are not owners or publishers and have no responsibility for its contents or policies.

See inside back cover or msp.org/pjm for submission instructions.

The subscription price for 2014 is US $\$ 410 /$ year for the electronic version, and \$535/year for print and electronic.

Subscriptions, requests for back issues and changes of subscribers address should be sent to Pacific Journal of Mathematics, P.O. Box 4163, Berkeley, CA 94704-0163, U.S.A. The Pacific Journal of Mathematics is indexed by Mathematical Reviews, Zentralblatt MATH, PASCAL CNRS Index, Referativnyi Zhurnal, Current Mathematical Publications and Web of Knowledge (Science Citation Index).

The Pacific Journal of Mathematics (ISSN 0030-8730) at the University of California, c/o Department of Mathematics, 798 Evans Hall \#3840, Berkeley, CA 94720-3840, is published twelve times a year. Periodical rate postage paid at Berkeley, CA 94704, and additional mailing offices. POSTMASTER: send address changes to Pacific Journal of Mathematics, P.O. Box 4163, Berkeley, CA 94704-0163.

PJM peer review and production are managed by EditFLOW ${ }^{\circledR}$ from Mathematical Sciences Publishers.

\section{PUBLISHED BY}

\section{mathematical sciences publishers \\ nonprofit scientific publishing}

http://msp.org/

(C) 2014 Mathematical Sciences Publishers 


\section{PACIFIC JOURNAL OF MATHEMATICS}

Volume $271 \quad$ No. $2 \quad$ October 2014

Monoids of modules and arithmetic of direct-sum decompositions

NiChOlas R. BAETH and AlFRED GEROLDINGER

On the torsion anomalous conjecture in $\mathrm{CM}$ abelian varieties

SARA CHECCOLI and EVELINA VIADA

Eigenvalue estimate and compactness for closed $f$-minimal surfaces

347

Xu Cheng, Tito Mejia and Detang Zhou

Lefschetz numbers of symplectic involutions on arithmetic groups

369

STEFFEN KIONKE

Categorification of a parabolic Hecke module via sheaves on moment 415 graphs

MARTINA LANINI

Unitary representations of $\operatorname{GL}(n, K)$ distinguished by a Galois

445 involution for a $p$-adic field $K$

NADIR MATRINGE

On $f$-biharmonic maps and $f$-biharmonic submanifolds

461

YE-LIN OU

Unitary principal series of split orthogonal groups

Alessandra Pantano, AnNegret Paul and Susana SALAMANCA RIBA 